\title{
Instabilities of a liquid film flowing down an inclined porous plane
}

\author{
Rong Liu and Qiusheng Liu* \\ Key Laboratory of Microgravity (National Microgravity Laboratory), Institute of Mechanics, Chinese Academy of Sciences, \\ Beijing 100190, China
}

(Received 13 January 2009; revised manuscript received 17 May 2009; published 29 September 2009)

\begin{abstract}
The problem of a film flowing down an inclined porous layer is considered. The fully developed basic flow is driven by gravitation. A careful linear instability analysis is carried out. We use Darcy's law to describe the porous layer and solve the coupling equations of the fluid and the porous medium rather than the decoupled equations of the one-sided model used in previous works. The eigenvalue problem is solved by means of a Chebyshev collocation method. We compare the instability of the two-sided model with the results of the one-sided model. The result reveals a porous mode instability which is completely neglected in previous works. For a falling film on an inclined porous plane there are three instability modes, i.e., the surface mode, the shear mode, and the porous mode. We also study the influences of the depth ratio $\hat{d}$, the Darcy number $\delta$, and the Beavers-Joseph coefficient $\alpha_{B J}$ on the instability of the system.
\end{abstract}

DOI: 10.1103/PhysRevE.80.036316

PACS number(s): 47.20.Ft, 47.15.gm, 47.56.+r, 47.11.Kb

\section{INTRODUCTION}

Falling film on an inclined plane has been a major problem in fluid mechanics for many years. The instability of falling film occurs in many physical systems and natural processes as well. Benjamin [1] and Yih [2] were the first to investigate the long-wave instability of a falling film on an impermeable inclined plane. Their results show that the long surface waves that propagate downstream at about twice the basic speed at the interface are insensitive to the velocity profile in the liquid and only weakly dependent on surface tension near the critical Reynolds number. Yih's results show that the falling film may be unstable to infinitesimal periodic perturbation, and critical conditions of the Reynolds number for the onset of instability have been obtained from the solution of the Orr-Sommerfeld equations. Benjamin's and Yih's results are only valid for small wave numbers. This instability mode is dependent on the interface deformation; in De Bruin's work [3] this long-wave surface mode is called the "soft mode." Lin [4] found a "hard mode" of instability, which is essentially a Tollmien-Schlichting shear wave in a flow with a free surface. Unfortunately, one of the boundary conditions is in error in [4]. De Bruin [3] pointed out the mistake in [4] and investigated the instability of corrected system in the case of zero surface tension. Floryan et al. [5] studied both the soft mode and the hard mode and paid particular attention to the effects of surface tension. Their results show that the characteristics of the hard mode depend on both the angle of inclination and the surface tension.

In the present paper, we are interested in the instabilities of a falling film flowing on a porous medium. The problems of flow instabilities of a fluid-porous system have a rich history. A careful look at previous works shows that instabilities of a fluid-porous system can be divided into two classes, i.e., the instabilities of thermal convection and the instabilities of parallel shear flows. Before we study the present problem, it is helpful to give a brief review of different instability modes

\footnotetext{
*liu@imech.ac.cn
}

occurring in a fluid-porous system. In a fluid-porous system, when instability occurs, the flows in the fluid and the porous medium display a variety of patterns. According to the structure of the flow pattern, the instabilities can be divided into two types. The first type is the fluid mode in which the flow cannot penetrate into the porous layer and is confined in the fluid layer. The second type is the porous mode in which the onset of instability occurs in both the fluid and the porous layers. These two instability modes can be triggered by either the temperature difference or the shear stress.

Early works on instability problems have concentrated on thermal convection in a fluid-porous system [6,7]. A good review of convection and heat transfer in porous medium can be referred in the work of Nield and Bejan [8]. More recently, the instability of parallel flow in a fluid-porous system has given rise to the interest of some investigators. Chang et al. [9] first addressed the problem of Poiseuille flow in a fluid-porous system in the framework of the Darcy model with a Beavers-Joseph interfacial condition [10]. The authors found that three modes of different stability characteristics may be triggered by the shear stress of the Poiseuille flow in such a system, i.e., the porous mode, the even-fluid-layer mode, and the odd-fluid-layer mode. Hill and Straughan [11] extended the work of [9] by introducing a Brinkman porous transition layer between the fluid layer and the Darcy-type porous medium and investigated the instability of the system. Liu et al. [12] studied instability of Poiseuille flow in a fluidporous system in the framework of the Brinkman model. Results in [12] show that no satisfactory agreement between Brinkman's model and Darcy's model is obtained for the instabilities of plane Poiseuille flow in a fluid-porous system. Both the results in $[11,12]$ indicate that if considering the effective viscosity of Brinkman's model, there are only two instability modes, i.e., the porous mode and the even-fluid layer mode, and the odd-fluid-layer mode disappears.

The instability of a falling film on an inclined porous layer is the extension of the problem of a falling film flowing on an impermeable wall. However, investigations on this problem are limited in previous works. To our knowledge, Pascal first [13] investigated the linear stability of a Newton- 


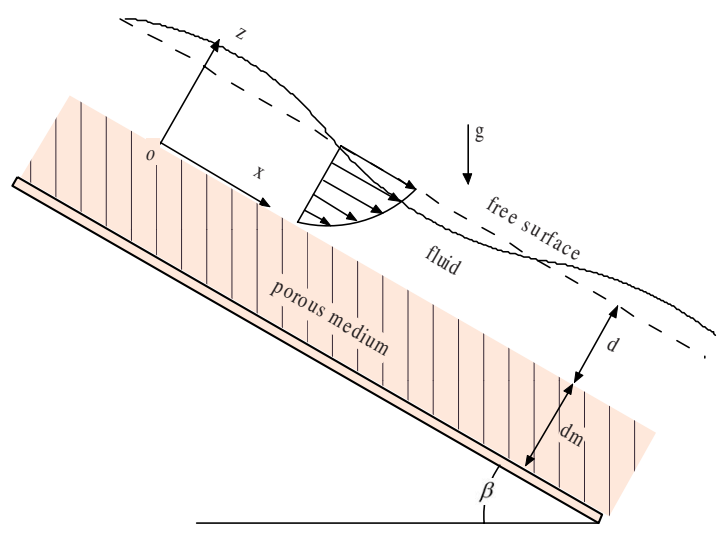

FIG. 1. (Color online) A sketch of the physical model.

ian fluid flowing down an inclined permeable plane. The author employed the same procedure proposed by Yin [2] and the results are only valid for long-wave region. In his work, Darcy's law together with the Beavers-Joseph condition is used to describe the flow in porous medium. However, the author made an assumption that $K / d^{2} \ll 1$ such that the flow in the porous medium can be neglected. Here $K$ is the permeability and $d$ is the thickness of the fluid film. Thus, the dynamics of the fluid layer and porous layer are decoupled. The effect of porous medium on the instability of the combined layers reduces to the influence of a slip boundary condition. This assumption was also used in Pascal's extended work [14] on the problem of instability of a power-law fluid flowing down an inclined porous plane. More recently, Sadiq and Usha [15] studied the linear and weakly nonlinear longwave stability of a thin Newtonian film flowing down a porous inclined plane. The authors used the same physical model with the same assumption as in Pascal's work $[13,14]$. However, a careful look at recent works on instability of Poiseuille flow in a fluid-porous system or a porous-fluidporous channel indicates that the filtration at the fluid-porous interface will play important roles in determining the instability mode. The negligence of the flow in porous layer sweeps the porous mode that occurs in the combined layers. In the present paper we considerably extend previous works by giving up the assumptions in [13] and solving the coupling equations of both the fluid and the porous medium rather than the one-sided equations of the fluid.

\section{PROBLEM FORMULATION}

Figure 1 illustrates the physical model of the present study. Consider a falling film of thickness $d$ on a porous plane of thickness $d_{m}$ inclined an angle $\beta$ to the horizontal direction. The coordinate $x$ is parallel to basic flow and $z$ is vertical to the inclined plane. The porous media are homogeneous and sealed by an impermeable wall at $z=-d_{m}$. The combined layers are infinite in the $x$ and $y$ directions. The fluid-porous interface is located at $z=0$ and the free surface of the fluid layer is at $z=d$. The surface tension $\sigma_{0}$ of the fluid interface is assumed to a constant. The free surface is a deformable interface described by $z=h(x, y, t)$, however, we assume that in basic state there is no surface deflexion. Thus, a fully developed, laminar flow is driven by gravitation, both in the fluid and the porous layers.

\section{A. Governing equations}

A classical approach to a fluid-porous system consists of solving the Navier-Stokes equations in the fluid and Darcy's equation in the porous medium. The Brinkman equation is an extension of Darcy's law. Two viscous terms appear in the Brinkman equation. The first is the usual Darcy term and the second is analogous to the Laplacian term that appears in the Navier-Stokes equation. The effect of the Laplacian term is not taken into accounted in Darcy's law. The Brinkman equation has been widely used to study flow in porous medium. An alternative approach to the present system is to apply the single-domain Brinkman formulation for both the liquid and the porous medium. In some recent works, the Brinkman model is also used in multidomain formulation. When using the Darcy model or the Brinkman model in multidomain formulation, the problem remains of defining relevant boundary conditions at the fluid-porous interface. Continuity of normal velocity is robust and generally accepted in most works. Until now, the definition of the boundary conditions relevant to pressure, tangential velocity, and shear stress remains an open question. In most works it is considered that the pressure has to be continuous across the interface. However, some authors argued that the total normal stress is continuous. In Nield's work [8], it is pointed that in practice, the viscous term may be small compared with the pressure, and in this case the continuity of total normal stress reduces to the approximate continuity of pressure. We find that using these two boundary condition leads to almost indistinguishable results. For the Darcy model, Beavers and Joseph [10] postulated a discontinuity in tangential velocity given by

$$
\frac{\partial u}{\partial z}=\frac{\alpha_{B J}}{\sqrt{K}}\left(u-u_{m}\right),
$$

where $\alpha_{B J}$ is the Beavers-Joseph coefficient, $z$ is the direction perpendicular to the interface, $u$ is the velocity of fluid, $u_{m}$ is the Darcy velocity inside the porous medium, and $K$ is the permeability. This boundary condition was validated experimentally by Beavers and Joseph. For the Brinkman equation, there is no general agreement regarding its conditions of applicability. The definition of its variable, especially the "effective" viscosity, and the boundary conditions seem to have some ambiguities. In some works, the continuities of tangential velocity and tangential stress have been used. However, these boundary conditions have not been justified by experiments.

In this paper, we assume that the flow through the porous medium satisfies Darcy's law. So, we use a same set of governing equations as most of previous works [6,9]. By setting the Darcy velocity to be zero, our model reduces to the onesided model in $[13,15]$.

For linear stability of the present problem, according to Squire's theorem [16] if a three-dimensional mode is unstable, a two-dimensional mode is unstable at a lower Reynolds number. So we only study the two-dimensional problem in this paper. In the fluid the governing equations are the 
continuity equation, the Navier-Stokes equations:

$$
\begin{gathered}
\frac{\partial u}{\partial x}+\frac{\partial w}{\partial z}=0, \\
\frac{\partial u}{\partial t}+u \frac{\partial u}{\partial x}+w \frac{\partial u}{\partial z}=-\frac{1}{\rho} \frac{\partial p}{\partial x}+g \sin \beta+\nu \nabla^{2} u, \\
\frac{\partial w}{\partial t}+u \frac{\partial w}{\partial x}+w \frac{\partial w}{\partial z}=-\frac{1}{\rho} \frac{\partial p}{\partial z}-g \cos \beta+\nu \nabla^{2} w .
\end{gathered}
$$

Here $u$ and $w$ are the velocities in the $x$ and $z$ directions, $p$ is the pressure, $g$ is the gravitation, $\beta$ is the inclined angle, $\nu$ is the viscosity, and $\nabla$ is the Laplacian.

The flow in the porous medium is described by the Darcy equation. The continuity equation and the Darcy equations are

$$
\begin{gathered}
\frac{\partial u_{m}}{\partial x}+\frac{\partial w_{m}}{\partial z}=0, \\
\frac{1}{\phi} \frac{\partial u_{m}}{\partial t}=-\frac{1}{\rho} \frac{\partial p_{m}}{\partial x}-\frac{\nu}{K} u_{m}+g \sin \beta, \\
\frac{1}{\phi} \frac{\partial w_{m}}{\partial t}=-\frac{1}{\rho} \frac{\partial p_{m}}{\partial z}-\frac{\nu}{K} w_{m}-g \cos \beta,
\end{gathered}
$$

where $\hat{d}$ and $w_{m}$ are the Darcy velocities of $x$ and $z$ directions, $p_{m}$ is the intrinsic volume average pressure, $\phi$ is the porosity, and $K$ is the permeability. For a porous medium consisting of glass spheres, $K$ is obtained by Kozeny-Carmen relation $[17]$

$$
K=\frac{D^{2}}{172.8} \frac{\phi^{3}}{(1-\phi)^{2}}
$$

in which $D$ is the diameter of the spheres.

At the bottom wall $z=-d_{m}$, there is no penetration

$$
w_{m}=0 \text {. }
$$

The boundary conditions at the free surface are the kinematic condition, the condition of zero shear stresses, and the condition that the normal stress balances the surface tension. These boundary conditions can be referred in [5]. At the free surface $z=d+h$, we have the kinematic condition

$$
w=\frac{\partial h}{\partial t}+u \frac{\partial h}{\partial x}
$$

where $h$ is the interfacial deflexion.

The shear stress balance condition is

$$
\frac{\partial w}{\partial x}+\frac{\partial u}{\partial z}=0
$$

and the normal stress balance condition is

$$
2 \mu \frac{\partial w}{\partial z}-p=2 \sigma_{0} H,
$$

where $\sigma_{0}$ is the surface tension, $\mu$ is the dynamic viscosity, and the mean curvature $H$ is defined by

$$
2 H=\frac{h_{x x}}{\left(1+h_{x}^{2}\right)^{3 / 2}} .
$$

At the fluid-porous interface $z=0$, the boundary conditions include the Beavers-Joseph interfacial condition Eq. (1), the continuity of the normal velocities

$$
w=w_{m},
$$

and the normal stress balance condition

$$
p-2 \mu \frac{\partial w}{\partial z}=p_{m}
$$

in which $\mu$ is the kinematic viscosity of fluid. We note that many works use the continuity of pressure. Here we use the same condition of continuity of normal stress as that in [18].

\section{B. Dimensionless equations and boundary conditions}

\section{Basic state}

For the fluid-porous system governed by Eqs. (2)-(7) and boundary conditions (1) and (9)-(15), we can find a steady basic solution. The lengths are normalized by $d$ for the fluid layer and $d_{m}$ for the porous layer. At this stage, the velocities in both layers are normalized by $u_{0}$ which is defined as

$$
u_{0}=g \sin \beta d^{2} / 2 \nu \text {. }
$$

The dimensionless controlling equations for basic velocity are

$$
\begin{gathered}
\frac{d^{2} \bar{u}}{d \bar{z}^{2}}=-2, \\
\bar{u}_{m}=2 \delta^{2} \hat{d}^{-2},
\end{gathered}
$$

with boundary conditions, at $\bar{z}=1$,

$$
\frac{d \bar{u}}{d \bar{z}}=0
$$

and at $\bar{z}=0$,

$$
\frac{d \bar{u}}{d \bar{z}}=\frac{\alpha_{B J} \hat{d}}{\delta}\left(\bar{u}-\bar{u}_{m}\right),
$$

where the depth ratio $\hat{d}=d / d_{m}$.

We obtain the dimensionless velocity in the fluid layer

$$
\bar{u}=-\bar{z}^{2}+2 \bar{z}+\frac{2 \delta}{\alpha_{B J} \hat{d}}+2 \frac{\delta^{2}}{\hat{d}^{2}}
$$

in which $\delta$ is the Darcy number defined as $\delta=\sqrt{K} / d_{m}$. The depth ration $\hat{d}$ is defined as $\hat{d}=d / d_{m}$. The fourth term of right-hand side in Eq. (21) is the longitudinal velocity in porous layer and the third term is the velocity slip at the fluid-porous interface. We use $\beta^{\prime}$ to denote the third term. Note that the fourth term is identical to $2\left(\frac{\sqrt{K}}{d}\right)^{2}$. The ratio of the fourth term to the third term is $\left(\frac{\sqrt{K}}{d}\right)^{2} / \beta^{\prime}=\alpha_{B J} \sqrt{K} / d$. In general $\alpha_{B J}$ is smaller than $\mathcal{O}(1)$, and under the assumption 
in Pascal's work $\sqrt{K} / d$ is a small number. So, the fourth term can be neglected and the third term should be retained in the case of $\left(\frac{\sqrt{K}}{d}\right)^{2} \ll 1$. This means the magnitude of the longitudinal velocity in the porous layer is much smaller than the velocity slip at the fluid-porous interface. Dropping the fourth term, Eq. (21) reduces to the basic solution in Pascal's work.

\section{Perturbed state}

We have chosen the maximum velocity of a film flowing on an impermeable plane $u_{0}=g \sin \beta d^{2} / 2 \nu$ as velocity scale for basic flow. It is convenient to choose this velocity as the scale for the fluid layer. For the porous layer, we will choose a difference set of scales. The scales of length, time, velocity, and pressure are $d, d / u_{0}, u_{0}$, and $\rho u_{0}^{2}$ in the fluid layer, and they are $d_{m}, d_{m}^{2} / \nu, \nu / d_{m}$, and $\rho\left(\nu / d_{m}\right)^{2}$ in the porous layer. From this stage, all the variables in the present study are in dimensionless form.

For the two-dimensional problem, we introduce the stream functions $\psi$ and $\psi_{m}$ by

$$
\begin{gathered}
u^{\prime}=\frac{\partial \psi}{\partial z}, \quad w^{\prime}=-\frac{\partial \psi}{\partial x}, \\
u_{m}^{\prime}=\frac{\partial \psi}{\partial z_{m}}, \quad w_{m}^{\prime}=-\frac{\partial \psi_{m}}{\partial x_{m}},
\end{gathered}
$$

where the prime denotes perturbational variables. We can further introduce the normal modes

$$
\begin{gathered}
\psi=\Phi(z) \exp (\sigma t+i k x), \\
\psi_{m}=\Phi_{m}\left(z_{m}\right) \exp \left(\sigma_{m} t_{m}+i k_{m} x_{m}\right) .
\end{gathered}
$$

Note that

$$
\begin{gathered}
\sigma=\sigma_{m} \frac{\hat{d}^{2}}{\operatorname{Re}}, \\
k=k_{m} \hat{d} .
\end{gathered}
$$

Here $k$ and $k_{m}$ are the wave numbers for the fluid and the porous layers, $\sigma$ and $\sigma_{m}$ are the complex time growth rates for these two layers, and Re is the Reynolds number defined as

$$
\operatorname{Re}=\frac{u_{0} d}{\nu} .
$$

The linearized amplitude equations and the boundary conditions take the form

$$
\begin{gathered}
\sigma \nabla^{2} \Phi+i k \bar{u} \nabla^{2} \Phi-i k \frac{d^{2} \bar{u}}{d z^{2}} \Phi=\frac{1}{\operatorname{Re}} \nabla^{2} \nabla^{2} \Phi, \\
\frac{1}{\phi} \sigma_{m} \nabla_{m}^{2} \Phi_{m}=-\frac{1}{\delta^{2}} \nabla_{m}^{2} \Phi_{m} .
\end{gathered}
$$

At $z_{m}=-1$,

$$
\Phi_{m}=0 .
$$

At the interface $z=z_{m}=0$ :

$$
\Phi=\frac{1}{\operatorname{Re}} \Phi_{m},
$$

$$
\begin{gathered}
\frac{1}{\operatorname{Re}} D^{3} \Phi-\frac{3 k^{2}}{\operatorname{Re}} D \Phi+i k \frac{d \bar{u}}{d z} \Phi-i k \bar{u} D \Phi+\frac{\hat{d}^{3}}{\delta^{2} \operatorname{Re}^{2}} D_{m} \Phi_{m} \\
=\sigma D \Phi-\sigma_{m} \frac{\hat{d}^{3}}{\phi \operatorname{Re}^{2}} D_{m} \Phi_{m} \\
D^{2} \Phi=\frac{\alpha_{B J} \hat{d}}{\delta}\left(D \Phi-\frac{\hat{d}}{\operatorname{Re}} D_{m} \Phi_{m}\right) .
\end{gathered}
$$

At $z=1$,

$$
\begin{gathered}
-i k \Phi=\sigma h+i k \bar{u} h, \\
D^{2} \Phi+k^{2} \Phi+\frac{d^{2} \bar{u}}{d z^{2}} h=0, \\
\operatorname{Re}^{-1} D^{3} \Phi+i k \frac{d \bar{u}}{d z} \Phi-i k \bar{u} D \Phi-\sigma D \Phi-3 \operatorname{Re}^{-1} k^{2} D \Phi \\
=\left(i k^{3} S+2 i k \operatorname{Re}^{-1} \cot \beta\right) h .
\end{gathered}
$$

Here $D=\frac{d}{d z}$ and $D_{m}=d / d z_{m}$. Note that $u_{0}=g \sin \beta d^{2} / 2 \nu$, the Reynolds number can also be expressed as

$$
\operatorname{Re}=\frac{g d^{3} \sin \beta}{2 \nu^{2}} .
$$

$S$ is the nondimensional surface tension defined as

$$
S=\frac{\sigma_{0}}{\rho u_{0}^{2} d} .
$$

We can also introduce the surface tension number as [5]; the surface tension number $\zeta$ is defined as

$$
\zeta=\left(\frac{3 \rho \sigma_{0}^{3}}{g \mu^{4}}\right)^{1 / 3} .
$$

Note that $\operatorname{Re}, S$, and $\zeta$ are not independent; they have the relation

$$
S=\operatorname{Re}^{-5 / 3}\left(\frac{3}{2} \sin \beta\right)^{-1 / 3} \zeta .
$$

\section{NUMERICAL METHOD}

We implement a Chebyshev collocation method to solve the eigenvalue problem. The domains of $(-1,0)$ and $(0,1)$ are transformed to the Chebyshev domain $(-1,1)$ by introduction of $\eta_{m}=2 z_{m}+1$ and $\eta=2 z-1$. The variables $\Phi$ and $\Phi_{m}$ are expanded as 


$$
\Phi=\sum_{n=0}^{N} \hat{\Phi}^{n} T_{n}(\eta), \quad \Phi_{m}=\sum_{n=0}^{N} \hat{\Phi}_{m}^{n} T_{n}\left(\eta_{m}\right) .
$$

Equations (29) and (30) together with corresponding boundary conditions lead to a sixth-order eigenvalue problem. Using Chebyshev series (42), the system of equations is required to solve for $2(N+1)+1$ unknowns including $2(N+1)$ spectral coefficients and an interface deflexion $h$. The details of imposing boundary conditions for spectral collocation method are described in $[12,19]$. This gives rise to a $(2 N+3) \times(2 N+3)$ matrix eigenvalue problem of the form

$$
A X=\sigma B X .
$$

In the present problem, the real part of the complex eigenvalue $\sigma_{r}$ is the time growth rate and the imaginary part $\sigma_{i}$ is the oscillatory frequency. Details of the computational procedure are described in [12].

We first check our computation procedure by comparing the eigenvalues with that of a liquid film flowing down an impermeable inclined plane [5]. We set the velocity in the porous layer to be zero and substitute the Beavers-Joseph boundary condition with a nonslip condition so that the coupling equations in the present paper reduce to that in [5]. We obtain the same results with that of [5].

Our code has also been used to solve the problem of Poiseuille flow in a fluid-porous system. In [12] we have compared our results with that of Chang et al. [9]. Our results are in good agreement with [9]. We have checked the convergence of our code. It was found that for small or moderate Re, $N=30$ yields satisfactory results. However, when $\operatorname{Re}>10000$ we need more items to expand the variable to obtain satisfactory results. In the present paper, we find that $N=60$ is enough to yield satisfactory results.

\section{RESULTS AND DISCUSSION}

Benjamin [1] and Yih [2] were the first to find the longwave surface instability for a liquid film flowing down an impermeable wall. De Bruin [3] found the shear mode of instability for such a system. It was found that for a large inclined angle $\beta$, the surface mode is more unstable than the shear mode. When the inclined angle $\beta$ is very small, the shear mode has a lower critical Reynolds number than that of the surface mode. Pascal $[13,14]$ and Sadiq et al. [15] only considered the surface mode, and in these works inclined angles are rather large, $\beta=45^{\circ}$. In these cases, the shear mode is completely dominated by the surface mode. In the works of De Bruin [3] and Floryan et al. [5], the inclined angles are rather small. In these cases, both the shear mode and the surface mode are very important. In the present paper, in order to compare the results of the two-sided mode to that of the one-sided model, we choose the same value of inclined angle as [15], i.e., $\beta=45^{\circ}$. When investigating the influences of parameters $\hat{d}, \delta$, and $\alpha_{B J}$ on different instability modes, we follow Floryan [5] and limit our analysis to the case of small inclined angle. In this case, it is convenient to compare the instability behaviors of the surface mode and the shear mode.

\section{A. Comparison between two-sided model and one-sided model}

Pascal [13] first studied the linear stability of a flowing film over an inclined porous plane using a one-sided model by assuming that $\left(\frac{\sqrt{K}}{d}\right)^{2} \ll 1$. We have checked our numerical code by comparing the critical Reynolds numbers with Pascal's results of long-wave analysis. A very good accord between our numerical results and Pascal's long-wave analysis is observed when $k \rightarrow 0$. However, for $k>0.2$ the instability curves of these two approaches present significant difference.

The depth ratio $\hat{d}$, the Darcy number $\delta$, and the BeaversJoseph constant $\alpha_{B J}$ are key parameters influencing the stability of the system. The dynamics of flow in porous layer is neglected in the one-sided model, of which the boundary condition indicates that the influences of these parameters are not independent. In $[13,15]$, a new parameter of the combination of $\hat{d}, \delta$, and $\alpha_{B J}$ is introduced to describe the influence of the porous layer on the instability of the system. In our notation, this parameter $\beta^{\prime}$ is defined as $\delta / \alpha_{B J} \hat{d}$.

Sadiq et al. [15] presented the critical Reynolds numbers for various $\beta^{\prime}$ from $0 \sim 0.4$ with an inclined angle $\beta=45^{\circ}$. In order to justify the validation of the one-sided model, we first compare the critical conditions of the two-sided model with that of the one-sided model of Pascal [13] and Sadiq et al. [15]. In Fig. 2, we present the critical Reynolds number of both the one-sided and the two-sided models in the $k-\mathrm{Re}$ plane for several typical cases of $\beta^{\prime}=0.1,0.2,0.3,0.4$ with $\beta=45^{\circ}$. Note that $\beta^{\prime}=\delta / \alpha_{B J} \hat{d}$. For a given value of $\beta^{\prime}$, we can change the values of $\delta$ and $\hat{d}$ by fixing $\alpha_{B J}=0.1$. We also note that $\left(\frac{\sqrt{K}}{d}\right)^{2}=\left(\alpha_{B J} \beta^{\prime}\right)^{2}$. The parameter $\left(\frac{\sqrt{K}}{d}\right)^{2}$ is in the range of 0.0001-0.0016. So, according to Pascal's assumption the one-sided model can be used for these parameters.

In Fig. 2(a), we present the marginal curves of the twosided model for $\delta=0.01,0.001$ and the marginal curves of the one-sided model. The critical values of these two models show almost no difference for $\beta^{\prime}=0.1$. For a larger $\beta^{\prime}=0.2$, the curve of $\delta=0.01$ of the two-sided model is indistinguishable from that of the one-sided model. The critical value of $\delta=0.001$ is slightly higher than that of the one-sided model. For $\beta^{\prime}=0.3,0.4$, the critical values of $\delta=0.001$ of the twosided model in the long-wave region are significantly higher than that of the one-sided model. In Fig. 2(d) for $\beta^{\prime}=0.4$, the critical value of the one-sided model is about $8 \%$ lower than that of $\delta=0.001$ of the two-sided model. Moreover, as shown in Fig. 2(d), the critical wave number of $\delta=0.001$ is obviously larger than that of the one-sided model of which the critical wave number is very close to be zero.

From the discussions above, it seems that the one-sided model somewhat underestimates the critical Reynolds number for the surface mode instability. For the surface mode of instability, the result of one-sided model is qualitatively valid in comparison with that of the two-sided model; however, it is accurate only when permeability is not very low or $\beta^{\prime}$ is small. We should note that in previous works, only the surface mode of instability has been investigated, so our comparison is only limited to the surface mode.

\section{B. Effects of the depth ratio $\hat{d}$}

The depth ratio $\hat{d}$ is a major parameter influencing the instability of a fluid-porous system. In the present work, we 

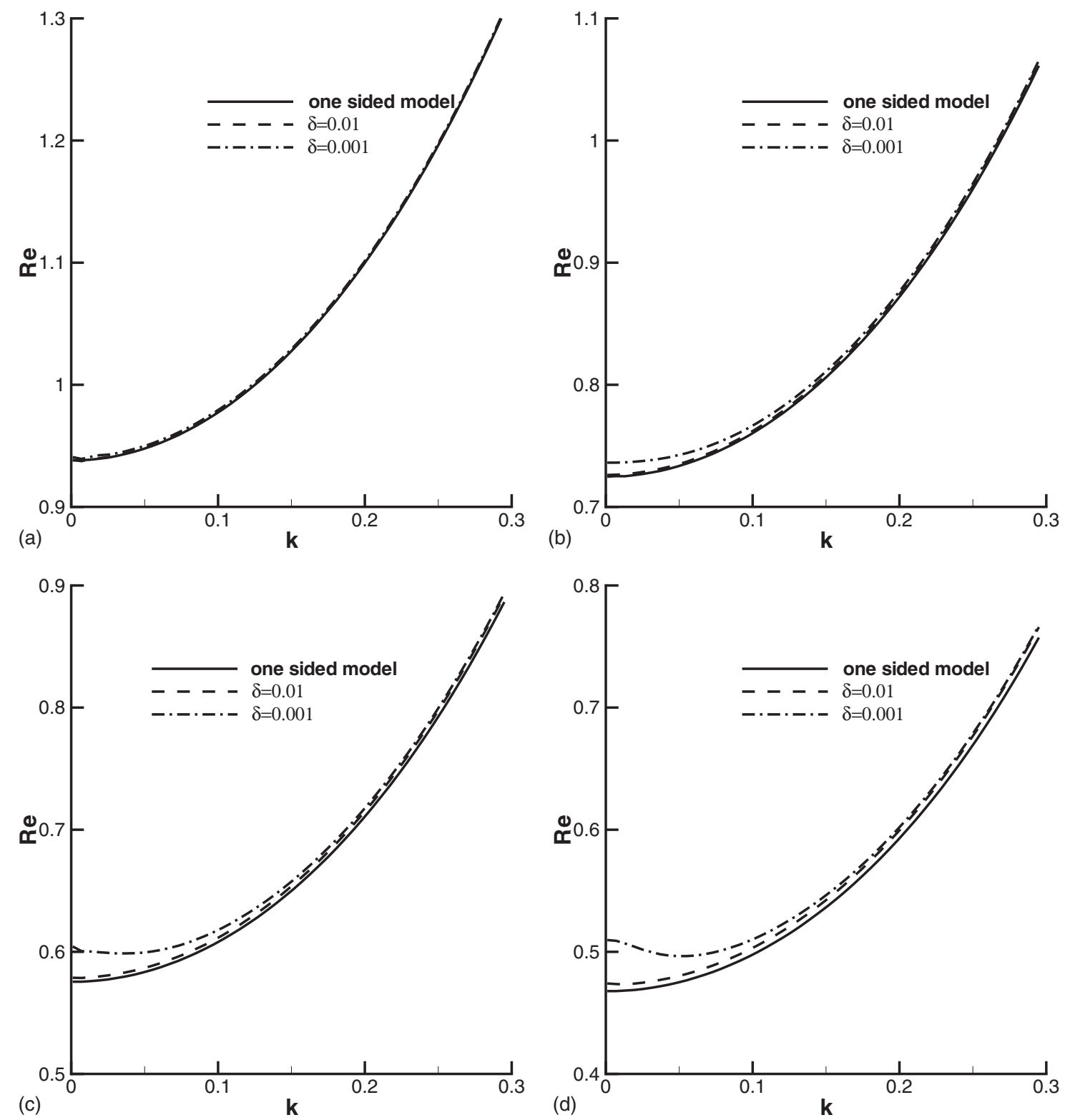

FIG. 2. Comparison of the marginal curves of the surface mode between the two-sided model and the one-sided model for various $\beta^{\prime}$. (a) $\beta^{\prime}=0.1$, (b) $\beta^{\prime}=0.2$, (c) $\beta^{\prime}=0.3$, and (d) $\beta^{\prime}=0.4$. The other parameters are $\beta=45^{\circ}$ and $\alpha_{B J}=0.1$.

will investigate the influence of $\hat{d}$ on different instability modes of a flowing film over a porous plane.

To characterize the effects of the depth ratio, we fix the parameters $\delta=0.001, \alpha_{B J}=0.1$ for some typical values of the inclined angle $\beta$. Figure 3 presents the marginal curves of the surface mode and the shear mode for various depth ratios $\hat{d}$ with inclined angles $\beta=4^{\circ}$ and $1^{\circ}$. For $\beta=4^{\circ}$ and $1^{\circ}$, the marginal curves display a bimodal structure. For each depth ratio, the upper branch is shear mode and the lower branch is surface mode. The marginal curves for $\hat{d}=1.0$ in Figs. 3(a) and 3(b) are qualitatively similar to that of a liquid film flowing on an impermeable wall presented in [5]. For the surface mode, we observe that with the decrease in $\hat{d}$ the critical Reynolds number decreases. This result is consistent with the result of the one-sided model that the critical Reynolds number decreases with the growth of $\beta^{\prime}$. For the shear mode, the influence of the depth ratio $\hat{d}$ on the instability characteristics is somewhat complicated. In Fig. 3(a), for $0.1 \leq \hat{d} \leq 1.0$ the unstable region of the shear mode in the $k-$ Re plane expands and the system becomes more unstable with the decrease in $\hat{d}$. For $\hat{d}<0.1$, the unstable region of the shear mode shrinks in the $k-$ Re plane with the decrease in $\hat{d}$. As $\hat{d}$ decreases to 0.02 , we find that the shear mode completely disappears in the range of $\mathrm{Re} \leq 10^{5}$.

In Fig. 3(b) for $\beta=1^{\circ}$, similar relation between the marginal curve of the shear mode and the depth ratio has also been observed. In Fig. 3, for each depth ratio $\hat{d}$ the surface branch predicts a lower value than that of the shear branch. So, on the onset of instability the surface mode is the dominant mode. The critical wave number of the dominant mode is $k_{c}=0$, where $c$ denotes the critical value. When the Reynolds number reaches $\mathrm{Re}_{c}$, the instability occurs in the form 


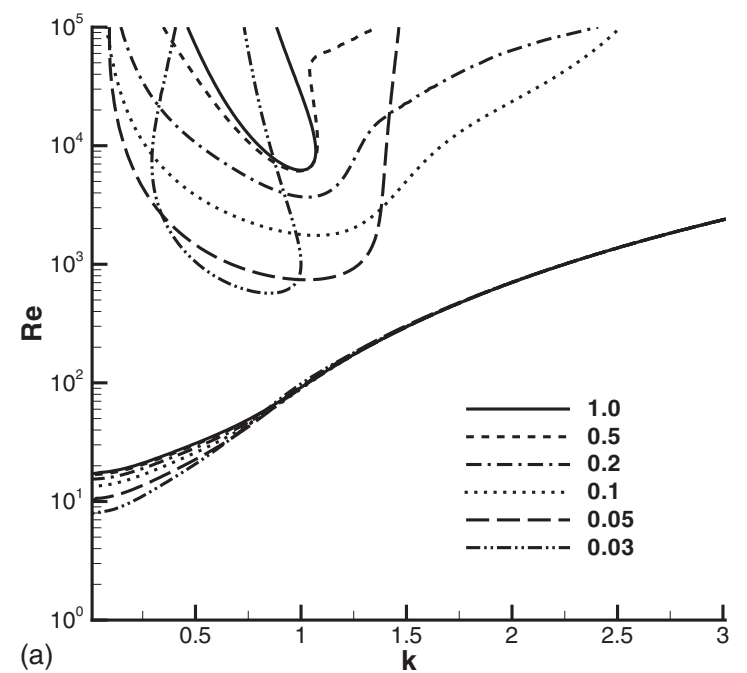

FIG. 3. The marginal curves for various depth ratios. (a) $\beta=4^{\circ}$ and

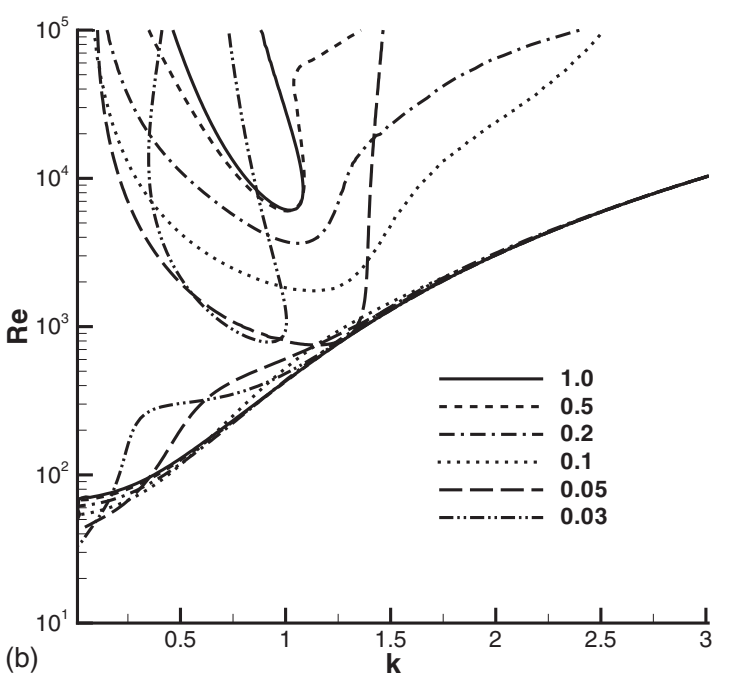

(b)

(b) $\beta=1^{\circ}$. The other parameters are $\delta=0.001$ and $\alpha_{B J}=0.1$. of long-wave surface mode. However, this does not mean that with the increase in the Reynolds number the surface mode is always the dominant. In order to understand the competition between the surface mode and the shear mode, we present the isolines of the scaled time growth rate in the $k-\operatorname{Re}$ plane for typical values of $\hat{d}=0.05$ with $\beta=4^{\circ}$ and $1^{\circ}$. We note that in the dimensionless equations the time scale is $d / u_{0}$. When comparing time growth rates for different Reynolds numbers, using $\sigma_{r}$ may lead to some confusion. Here it is convenient to rescale the time growth rate and use $\sigma_{r} \operatorname{Re}$ to compare the instability for different Reynolds numbers.

In Fig. 4(a), for a small Reynolds number the surface mode is the dominant mode. For a fixed wave number $k$ the time growth rate of the surface mode increases with Re. When Re increases to about 1000, it is found that the shear mode instability is excited with a time growth rate $\sigma_{r} \mathrm{Re}$ slightly higher than 8.34; however, the maximum time

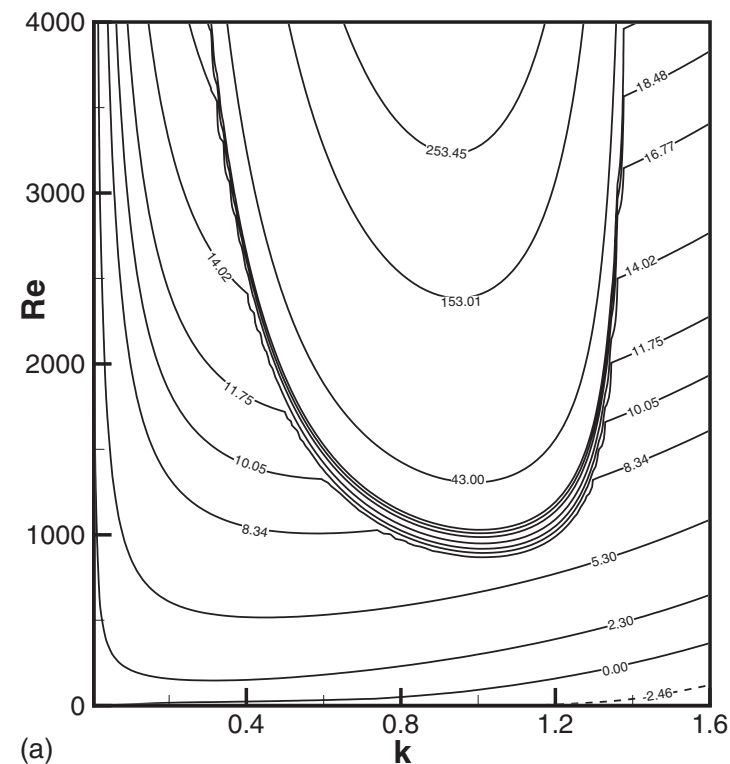

growth rate of the surface mode is slightly lower than 8.34. For $\operatorname{Re}>1000$, the shear instability becomes the dominant mode. When Re increases to a rather high value, it is obvious that the surface mode is completely suppressed by the shear mode. In Fig. 4(b), we have also observed the switch from the surface mode to the shear mode with the increase in Re.

In normal mode expansion, the perturbation consists of series of waves with different wave numbers. Investigating the effects of $\mathrm{Re}$ on the instability behavior of different modes with a fixed wave number is helpful to understand the competition between different modes.

Figures 5(a) and 5(b) illustrate the trajectories of eigenvalues of the phase velocity defined as $c=i \sigma / k$ as $\operatorname{Re}$ decreases from 10000 to 10 for parameters fixed at $k=1.0, \beta$ $=4^{\circ}, \hat{d}=0.2, \delta=0.001$, and $\alpha_{B J}=0.1$. In Fig. 5, $c_{r}$ and $c_{i}$ denote the real part and the imaginary part of $c$. The circled eigenvalues correspond to $\mathrm{Re}=10$ 000. The shear mode la-

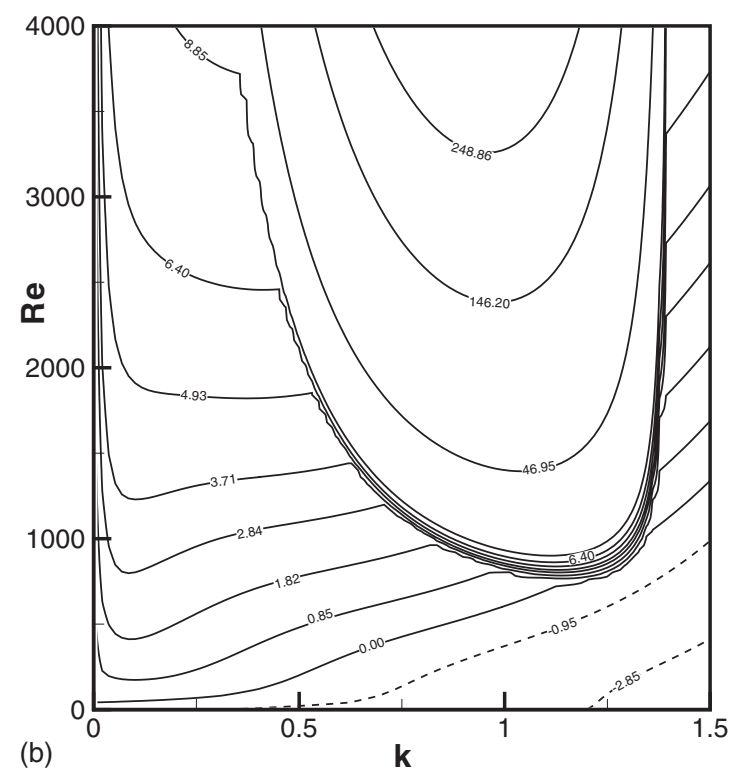

FIG. 4. The isolines of the rescaled time growth rate $\sigma_{r} \operatorname{Re}$ in the $k-\operatorname{Re}$ plane. (a) $\beta=4^{\circ}$ and (b) $\beta=1^{\circ}$. The other parameters are $\hat{d}=0.05, \delta=0.001$, and $\alpha_{B J}=0.1$. 

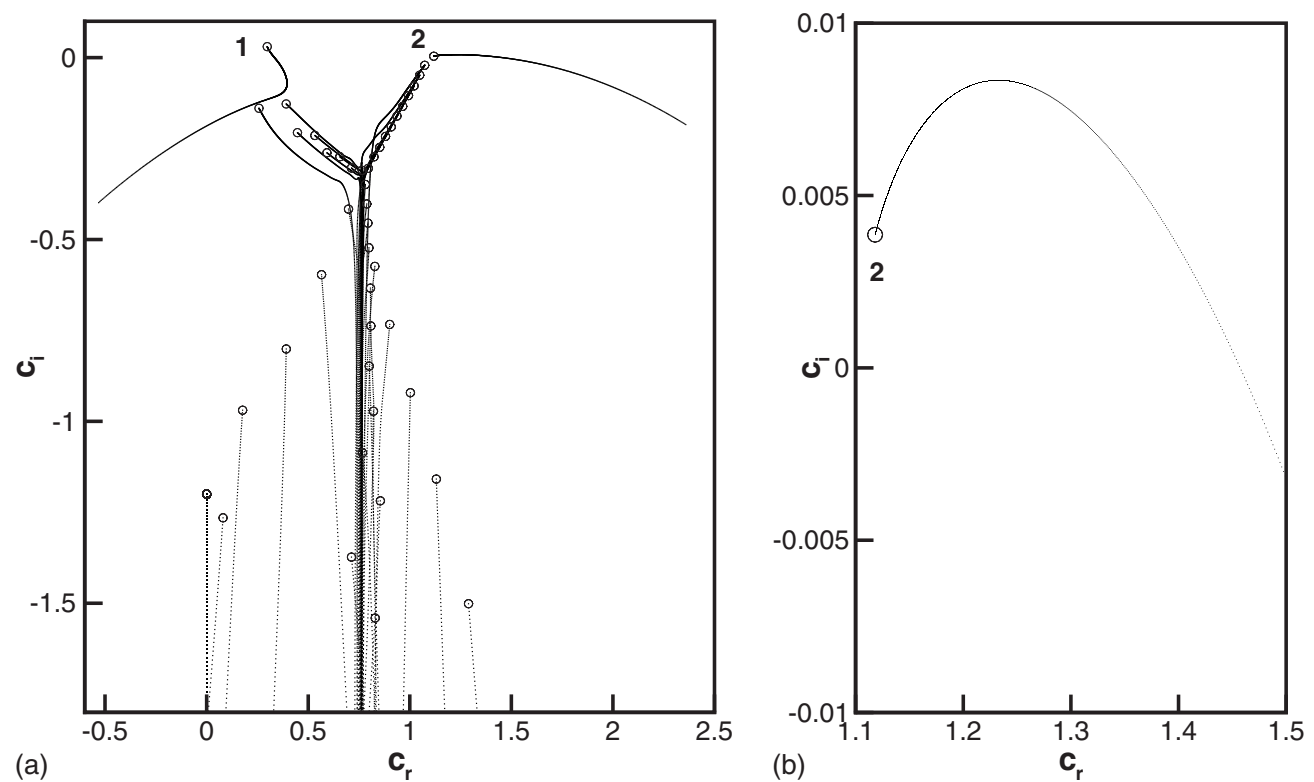

FIG. 5. The trajectories of the phase speeds $c$ with $k=1.0$ as Re decreases from 10000 to 10 . The other parameters are $\beta=4^{\circ}, \hat{d}=0.2$, $\delta=0.001$, and $\alpha_{B J}=0.1$.

beled 1 locates on the left branch and the surface mode labeled 2 on the right branch. For large Reynolds numbers, these two modes are unstable with $c_{i}>0$. As shown in Fig. 5, except the dominant mode 1 and mode 2, other modes are highly dampened with the decrease in Re. The trajectory of mode 1 shows that the shear mode is dampened less than the other modes. The magnified trajectory of mode 2 shows that with the decrease in $\mathrm{Re}, c_{i}$ of mode 2 increases at $\mathrm{Re}$ $=10000$ and reaches the maximum at a smaller Re. When Re decreases further, $c_{i}$ decreases with $\mathrm{Re}$ and the surface mode becomes a stable mode.

In Figs. 6(a) and 6(b), the marginal curves of $\beta=4^{\prime}$ are presented for various depth ratios. In comparison with the curves of $\beta=4^{\circ}$ and $1^{\circ}$, the branches of surface mode of $\beta=4^{\prime}$ in Figs. 6(a) and 6(b) are more stable than that in Fig. 3. In Figs. 6(a) and 6(b), for each depth ratio the surface branch and the shear branch intersect in medium wave number region. In Fig. 6(a), the surface branch of $\hat{d}=1.0$ is a monotonic function of $k$. When $\hat{d}$ decreases to 0.2 , a relative minimum enters the right part of the surface branch and this branch becomes steeper in the long-wave region. In Fig. 6(a) for $\hat{d} \geq 0.1$, it is obvious that with the decrease in $\hat{d}$ the shear branch expands in the $k-\operatorname{Re}$ plane. However, for $\hat{d} \leq 0.08$ in Fig. 6(b) the shear mode branch shrinks in the $k-\operatorname{Re}$ plane with the decrease in $\hat{d}$. When $\hat{d}$ decreases to 0.04 , we find that a bubble-shaped branch enters the long-wave region. In Fig. 6(b), with the decrease in $\hat{d}$, this new branch becomes more unstable and expands in the $k-$ Re plane. At this stage, we have not known the nature of the new branch. The marginal curves of $\hat{d}=0.04$ and 0.035 display a trimodal structure, i.e., the surface mode branch, the shear mode branch, and the bubble-shaped branch. Since the bubble-shaped branches locate in the long-wave region, the following question should be asked. Does this branch correspond to a new instability mode or is it only the deformation of the surface branch?
The marginal curves of $\beta=1^{\prime}$ and $6^{\prime \prime}$ are represented in Figs. 6(c)-6(f) for various depth ratios. We find that the influences of the depth ratio on the shear branch for $\beta=1^{\prime}$ and $6^{\prime \prime}$ are similar to that for $\beta=4^{\circ}$ and $1^{\circ}$. For $\hat{d} \geq 0.1$, as shown in Figs. 6(c) and 6(e) with the decrease in $\hat{d}$ the shear branches expand in the $k-\operatorname{Re}$ plane. For $\hat{d}<0.1$ as shown in Figs. 6(d) and 6(f), the shear branches shrink in the $k-\operatorname{Re}$ plane. In Fig. 6(d), when $\hat{d}$ decreases to 0.08 , we also find a bubble-shaped branch and the marginal curves for small depth ratios also display a trimodal structure. In Figs. 6(e) and 6(f), in the range of $\operatorname{Re}<100000$, the surface mode branch disappears. As $\hat{d}$ decreases to 0.2 , we find a new branch in the long-wave region. In Figs. 6(b), 6(d), and 6(f), we find that the decrease in $\hat{d}$ destabilizes the new long-wave branch.

In order to understand the physics of different instability modes in Fig. 6, we present the amplitudes of the stream functions of the shear mode in Fig. 7 and that of the new long-wave branch in Fig. 8. In Fig. 7, the amplitudes of $\Phi$ and $\Phi_{m}$ of shear mode are represented for three typical cases. It is obvious that the flow is confined in the fluid layer. This result indicates that the shear mode is a fluid mode. In Fig. 8, the amplitudes of $\Phi$ and $\Phi_{m}$ of the new long-wave branch are represented for three typical cases. In comparison with Fig. 7, the flow of this new branch penetrates to the porous layer and the circulation occurs in the combined layers. This means that the new long-wave branches in Fig. 6 are of different stability characteristics to the surface mode and the shear mode. In this paper, we refer to this mode as a porous mode.

Figure 9 illustrates the trajectories of the eigenvalues of the phase velocity as Re decreases from 100000 to 1000 , for $k=1.5, \beta=6^{\prime \prime}, \hat{d}=0.1, \delta=0.001$, and $\alpha_{B J}=0.1$. The circled eigenvalues correspond to $\mathrm{Re}=100000$. The shear mode and the porous mode are labeled 1 and 2 . As Re decreases from 

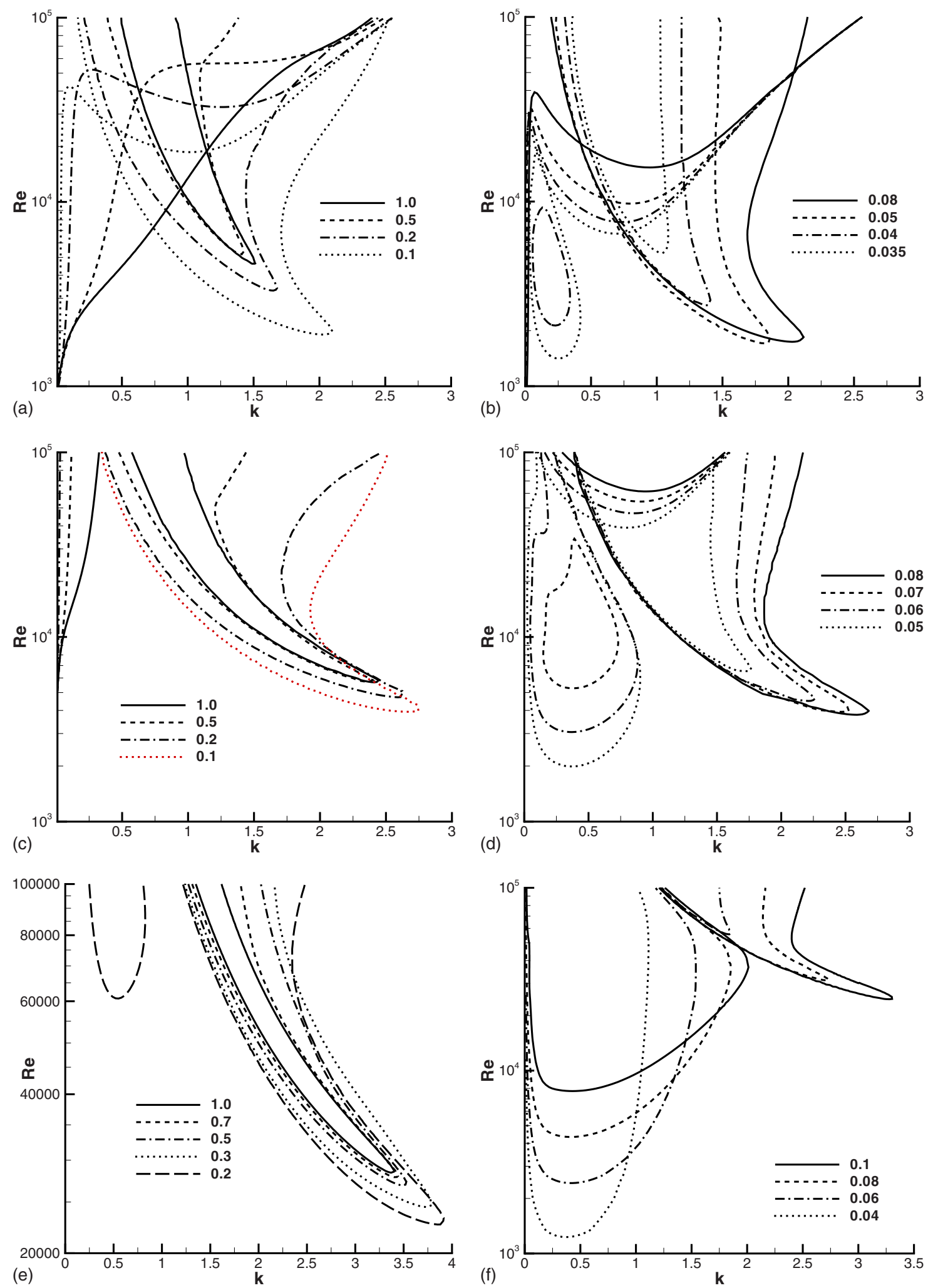

FIG. 6. (Color online) The marginal curves for various depth ratios. [(a),(b)] $\beta=4^{\prime},[(\mathrm{c}),(\mathrm{d})] \beta=1^{\prime}$, and $[(\mathrm{e}),(\mathrm{f})] \beta=6^{\prime \prime}$. The other parameters are $\delta=0.001$ and $\alpha_{B J}=0.1$.

100000 to 1000 , most of the modes are highly dampened. For $\mathrm{Re}=100000$ the shear mode is the dominant mode. The trajectory labeled 1 shows that for large Re the shear mode is highly dampened with the decrease in Re. As Re decreases further, the shear mode does not dampen with the decrease in Re. For lower Re, the decrease in Re only influences the frequency of the shear mode. For $\mathrm{Re}=100000$, the porous mode is stable. With the decrease in $\mathrm{Re}$, the porous mode 


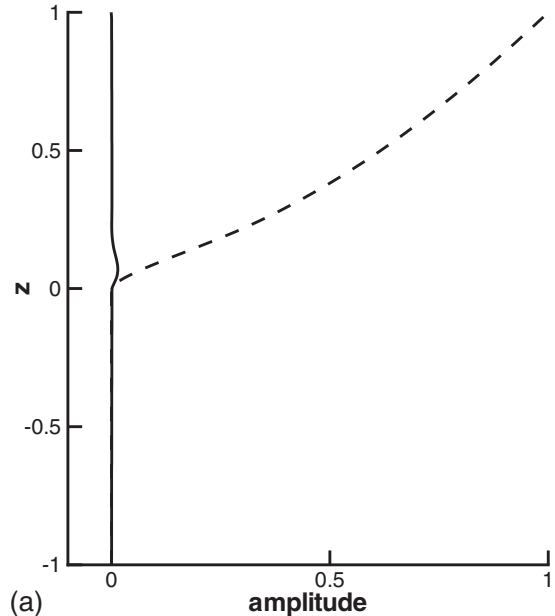

(a)

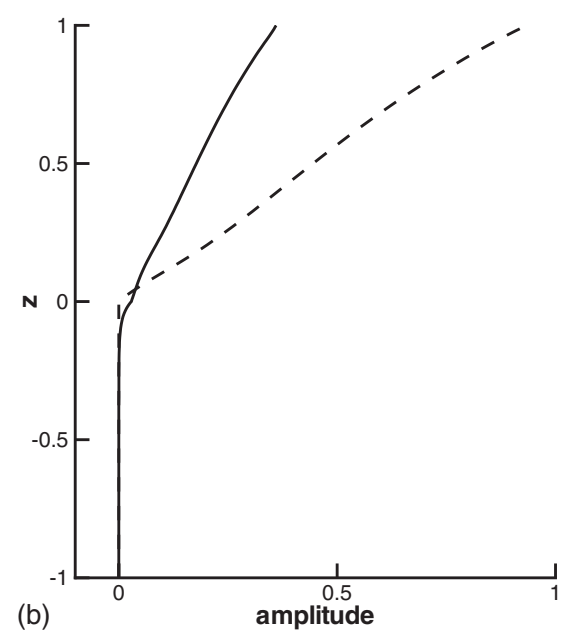

(b)

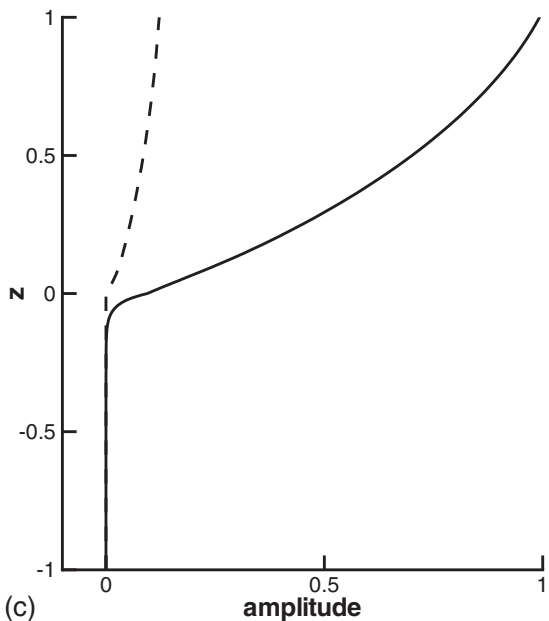

FIG. 7. The normalized amplitudes of $\Phi$ and $\Phi_{m}$ of the shear mode at critical states. The solid lines represent the real parts and the dashed lines represent the imaginary parts. (a) $\hat{d}=1.0, k=1.52$, and $\operatorname{Re}=4374$; (b) $\hat{d}=0.1, k=2.0$, and $\operatorname{Re}=1887$; (c) $\hat{d}=0.035, k=1.02$, and $\operatorname{Re}=5187$. The other parameters are $\beta=4^{\prime}, \delta=0.001$, and $\alpha_{B J}=0.1$.

becomes less stable. With the decrease in Re the trajectory labeled 2 protrudes into the unstable plane and the porous mode becomes the dominant mode. When Re decreases further, this mode begins to be dampened as other modes.

In order to understand the competition between the shear mode and the porous mode, we present the isolines of the rescaled time growth rate in the $k-\operatorname{Re}$ plane for two typical cases in Fig. 10. For $\beta=1^{\prime}, \hat{d}=0.05$ as shown in Fig. 10(a), the porous branch and the shear branch are separated in the $k-$ Re plane. The critical Reynolds number of the porous mode is obviously lower than that of the shear mode. In this figure, we find that the maximum time growth rate of the porous mode locates in the region near $k=0.4, \operatorname{Re}=15000$. This indicates that for $\operatorname{Re}<15000$ the increase in $\mathrm{Re}$ will destabilize the porous mode and for $\operatorname{Re}>15000$ the increase in Re will stabilize this mode. However, for the shear mode, the rescaled time growth rate $\operatorname{Re} \sigma_{r}$ increases with Re. In Fig. $10(\mathrm{a})$, it is apparent that the marginal curve of the porous mode is lower than that of shear mode. As Re increases to about 11000 , the time growth rates of these two modes are about $2.51 \times 10^{2}$. As Re increases further, the time growth rate of the shear mode becomes larger than that of the porous mode. As discussed above, the isolines predict that with the increase in $\mathrm{Re}$ the porous mode will switch to the shear mode.

For $\beta=6^{\prime \prime} \hat{d}=0.1$, as shown in Fig. $10(\mathrm{~b})$, the porous branch and the shear branch merge near the region $k=2.0$. With the increase in Re, such a switch from the porous mode to the shear mode has also been found.

\section{Effects of the Darcy number $\delta$}

The permeability plays an important role in determining the instability of the system. The Darcy number defined as $\delta=\sqrt{K} / d_{m}$ accounts for the permeability of the porous medium. For real porous medium, $K$ is in the range of

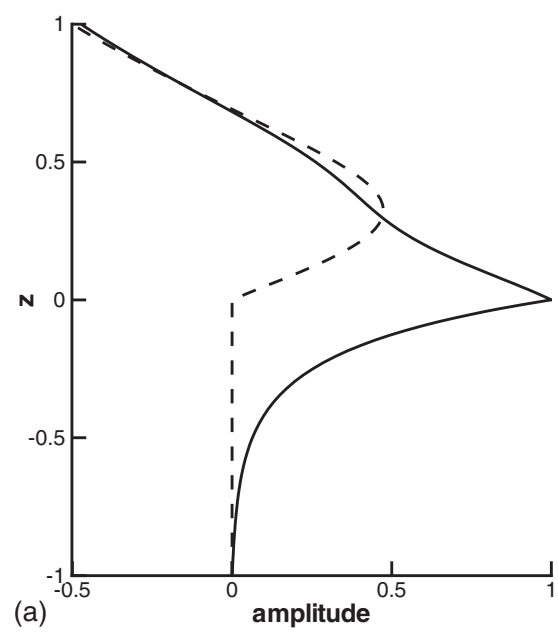

FIG. 8. The normalized amplitudes of $\Phi$ and $\Phi_{m}$ of the porous mode at critical states. The solid lines represent the real parts and the

dashed lines represent the imaginary parts. (a) $\hat{d}=0.04, k=0.22, \operatorname{Re}=2129$, and $\beta=4^{\prime}$; (b) $\hat{d}=0.2, k=0.53, \operatorname{Re}=60720$, and $\beta=6$; ; (c) $\hat{d}=0.04, k=0.40, \operatorname{Re}=1196$, and $\beta=6^{\prime \prime}$. The other parameters are $\delta=0.001$ and $\alpha_{B J}=0.1$.

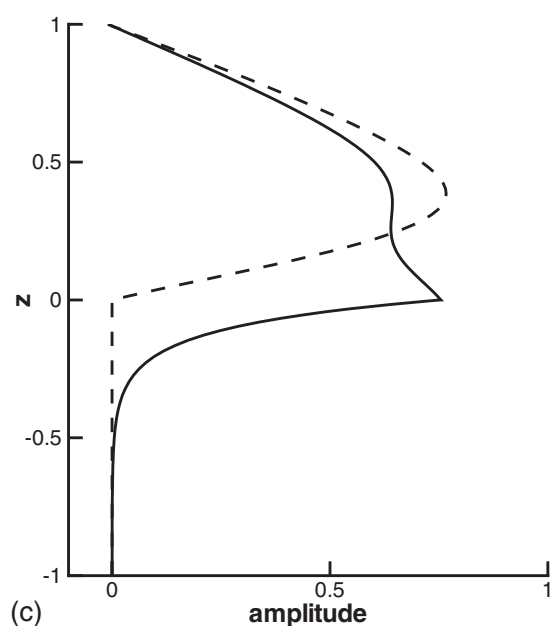

(c)

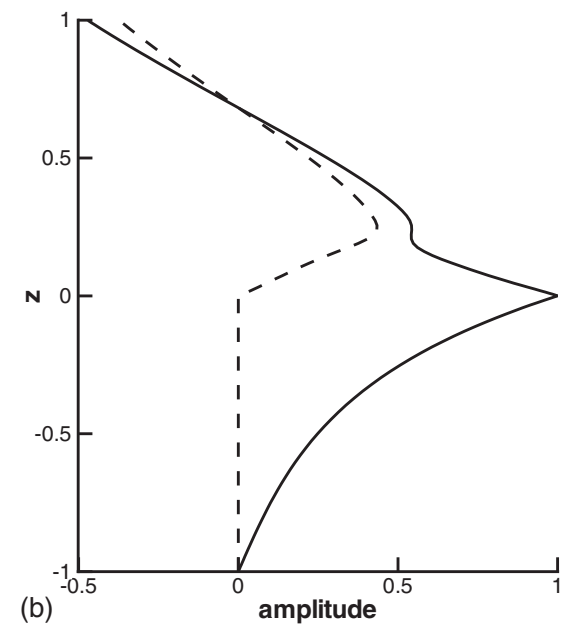




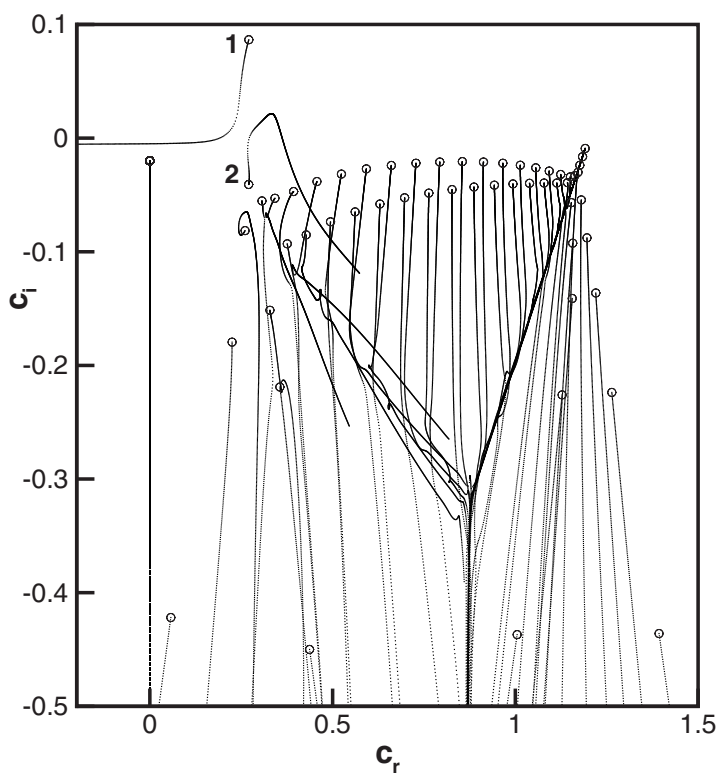

FIG. 9. The trajectories of the phase speeds $c$ for $k=1.5$ as Re decreases from 100000 to 1000 . The other parameters are $\beta=6$ ", $\hat{d}=0.1, \delta=0.001$, and $\alpha_{B J}=0.1$.

$10^{-15}-10^{-8} \mathrm{~m}^{2}$ [8]. In most applications, $d_{m}$ will be considerably greater than $\sqrt{2 K}$. In our computation, the Darcy number in the range of $10^{-4}-10^{-2}$ is physically realistic.

In order to characterize the effect of the permeability on the instability, we fix other parameters and vary only the Darcy number. In Fig. 11, the marginal curves for $\beta=4^{\circ}$, $\hat{d}=0.2$ and $\beta=6^{\prime \prime}, \hat{d}=0.1$ are presented. For $\beta=4^{\circ}, \hat{d}=0.2$ as shown in Fig. 11(a), the surface mode becomes more unstable in the long-wave region as $\delta$ increases from 0.0002 to 0.007 . In the short wave region, the surface mode is insensitive to $\delta$. This result is consistent to that of the one-sided model. With the increase in $\delta$, the marginal curve of the

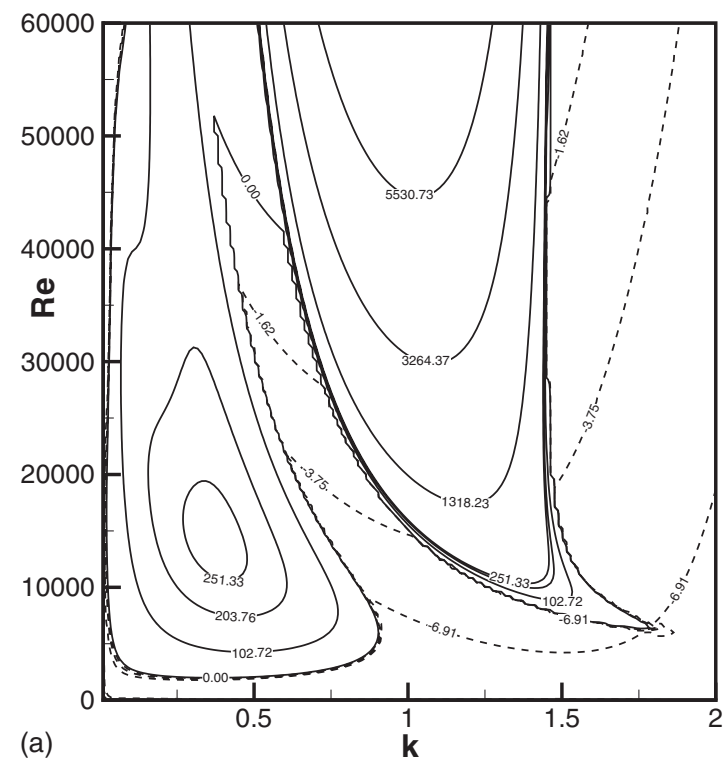

shear mode expands and then shrinks in the $k-$ Re plane. It seems that for low permeability case, the increase in $\delta$ significantly destabilizes the flow. However, for high permeability case the increase in $\delta$ stabilizes the shear mode.

In Fig. 11(b), in the range of $\operatorname{Re} \leq 10^{5}$ the marginal curve of $\delta=0.0002$ only has a shear branch. For $\delta=0.0005$, a porous branch presents in the long-wave region. For these two cases, owing to a very small $\delta$ (a very low permeability) in the porous medium, the flow is difficult to penetrate into the porous layer and the circulation is confined in the fluid layer. As a result, the porous mode is more stable than the shear mode. As shown in this figure, with the increase in $\delta$ the critical value of the porous mode significantly decreases. For $\delta<0.001$, with the increase in $\delta$ the porous branch expands in the $k-$ Re plane; for $\delta \geq 0.001$, with the increase in $\delta$ the porous mode shrinks. Physically speaking, owing to a higher permeability, the flow can more easily penetrate into the porous layer so that the porous mode instability becomes more unstable.

To gain more insight into the influence of permeability on the instability of the system, we present the trajectories of spectrums for a wave with $k=1.0, \operatorname{Re}=100000, \beta=4^{\circ}$, and $\hat{d}=0.2$ in Fig. 12 and with $k=0.5, \operatorname{Re}=100000, \beta=6^{\prime \prime}$, and $\hat{d}=0.1$ in Fig. 13 as the Darcy number varies from 0.0001 to 0.01. The circled points in Figs. 12 and 13 correspond to the spectrums of $\delta=0.0001$. In Fig. 11(a), the point $k=1.0$, $\operatorname{Re}=100000$ in the $k-\operatorname{Re}$ plane is near the marginal boundary of the curve of $\delta=0.0002$. In Fig. 11(a), for $\delta \leq 0.002$, with the increase in $\delta$ the marginal curve expands in the $k-\operatorname{Re}$ plane so that this point locates in the unstable region. In Fig. 12 the corresponding $c_{i}$ of the shear mode (labeled 1) increases and this mode becomes the dominant mode. In Fig. 11(a), for $\delta \geq 0.005$, with the increase in $\delta$ the shear branch shrinks in the $k-$ Re plane with the critical Reynolds number increasing and the critical wave number decreasing. For $\delta=0.007$, it is interesting that the shear branch encloses in

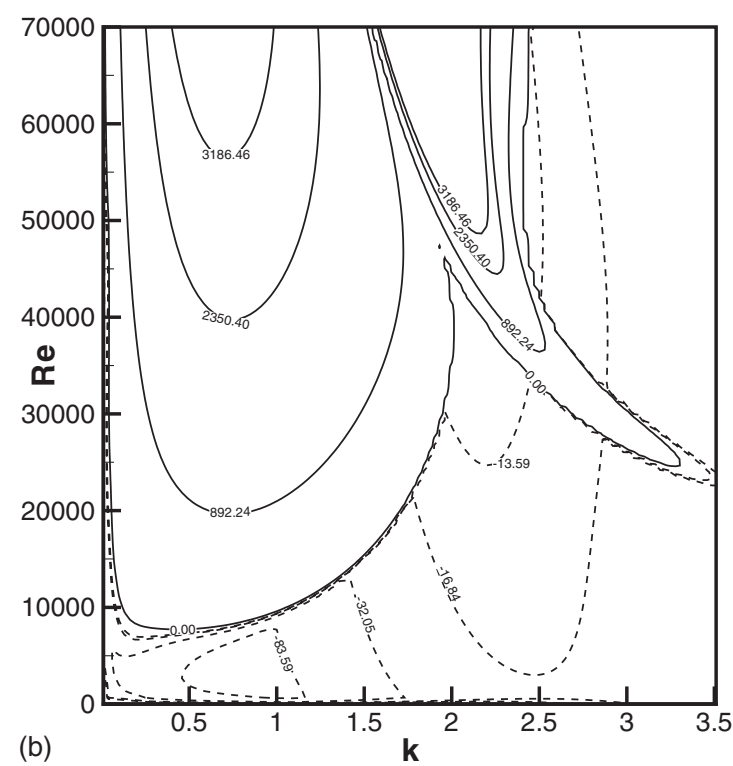

FIG. 10. The isolines of the rescaled time growth rate $\sigma_{r} \operatorname{Re}$ in the $k-\operatorname{Re}$ plane. (a) $\beta=1^{\prime}, \hat{d}=0.05$; (b) $\beta=6^{\prime \prime}, \hat{d}=0.10$. The other parameters are $\delta=0.001$ and $\alpha_{B J}=0.1$. 


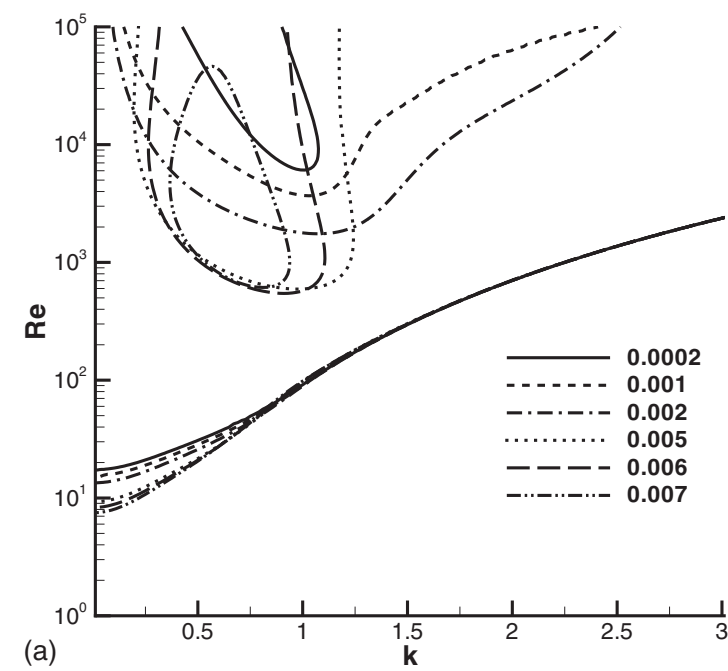

FIG. 11. The marginal curves for various Darcy numbers.

the $k-\operatorname{Re}$ plane. When $\delta$ increases more, the shear branch disappears. The corresponding trajectory in Fig. 12 shows that when $\delta$ exceeds a certain value the shear mode is dampened; as a result, $c_{i}$ of the shear mode becomes smaller than the leading mode of the right branch.

Figure 13 represents the trajectories of spectrums of $k$ $=0.5, \operatorname{Re}=10000, \beta=6^{\prime \prime}, \hat{d}=0.1$, and $\alpha_{B J}=0.1$ as $\delta$ varies from 0.0001 to 0.1 . The trajectory of the porous mode is labeled 1. In Fig. 13, with the increase in $\delta$ from 0.0001 to a certain value, $c_{i}$ increases. As $\delta$ increases more, $c_{i}$ of the porous mode significantly decreases. In order to understand the behavior of the trajectory of porous mode in Fig. 13, we repeat to analyze the marginal curves in Fig. 11(b). For $k$ $=0.5$, with the increase in $\delta$ from 0.001 , the difference between $k=0.5$ and the critical wave $k_{c}$ of the porous mode increases. The trajectory labeled 1 in Fig. 13 indicates that $c_{i}$

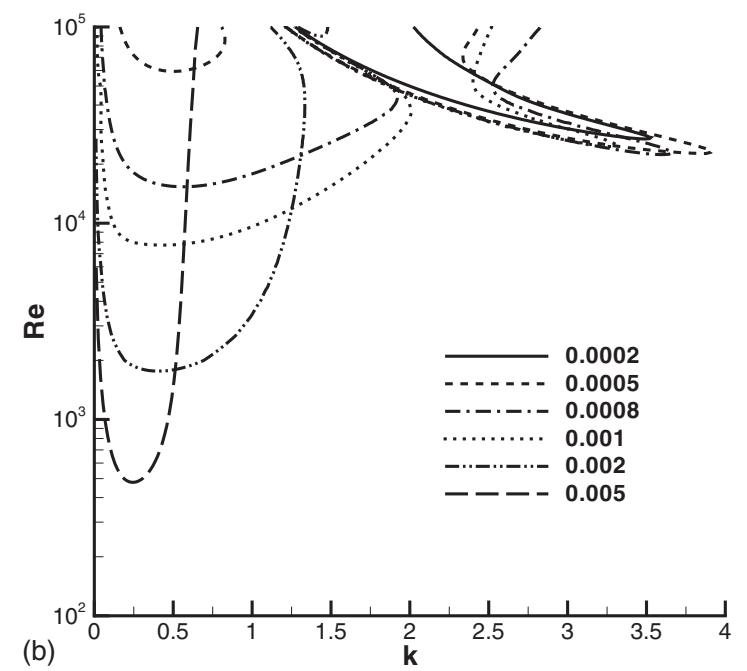

(a) $\beta=4^{\circ}, \hat{d}=0.2$, and $\alpha_{B J}=0.1$; (b) $\beta=6^{\prime \prime}, \hat{d}=0.1$, and $\alpha_{B J}=0.1$.

decreases with the increase in the magnitude of $k-k_{c}$.

In Figs. 12 and 13, it is found that for low permeability some modes become less stable with the increase in $\delta$. For high permeability, the frequencies of most of the modes increase with $\delta$; however, the imaginary parts of the time growth rates of most modes in the middle and the right branches are insensitive to a large $\delta$.

\section{Effects of the Beavers-Joseph constant $\alpha_{B J}$}

In the Beavers-Joseph condition, the dimensionless coefficient $\alpha_{B J}$ characterizes the structure of the permeable material near the fluid-porous interface and must be determined for each particular system. In Beavers and Joseph's work [1], $\alpha_{B J}$ has the values of $0.78,1.45$, and 4.0 for Foametal having average pore sizes $0.016,0.034$, and 0.045 in., respectively,
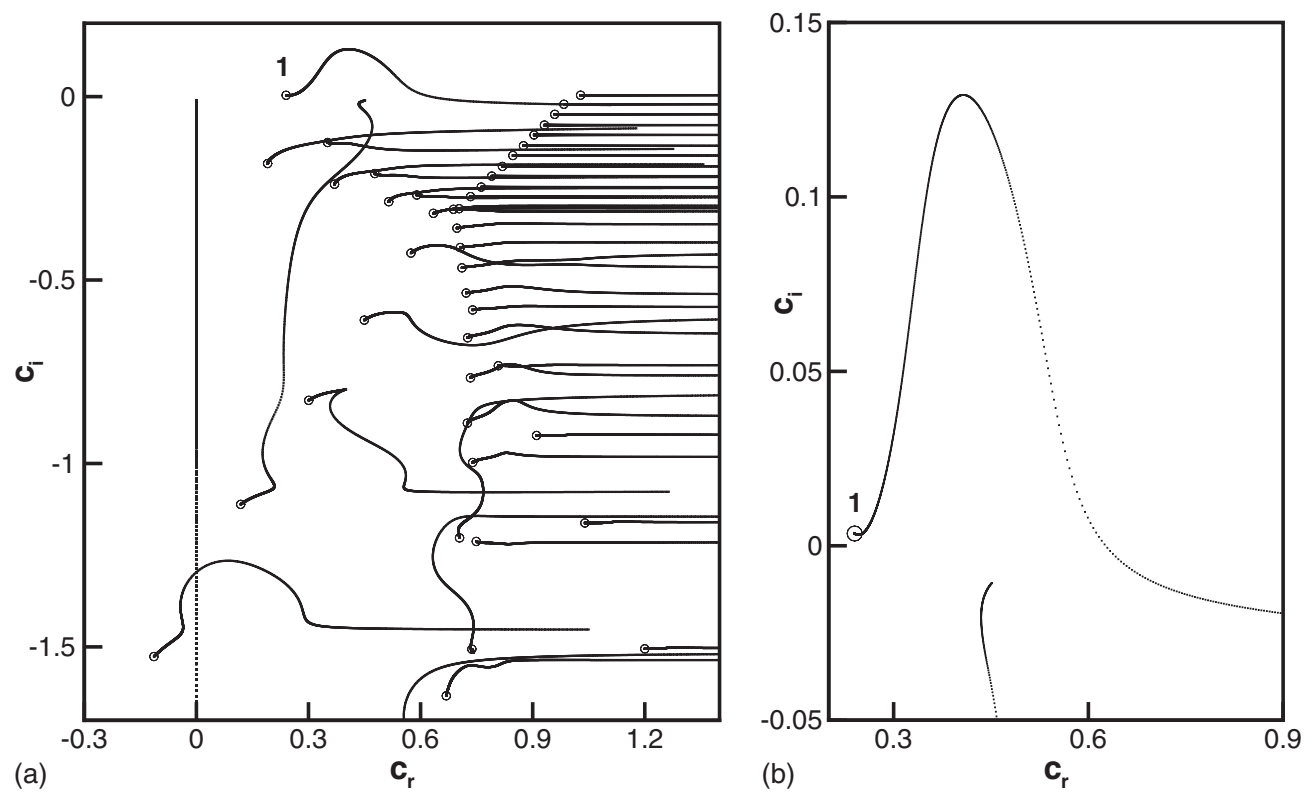

FIG. 12. The trajectories of the phase speeds $c$ for $k=1.0, \mathrm{Re}=10000$ as $\delta$ increases from 0.0001 to 0.01 . The other parameters are $\beta=4^{\circ}, \hat{d}=0.2$, and $\alpha_{B J}=0.1$. 

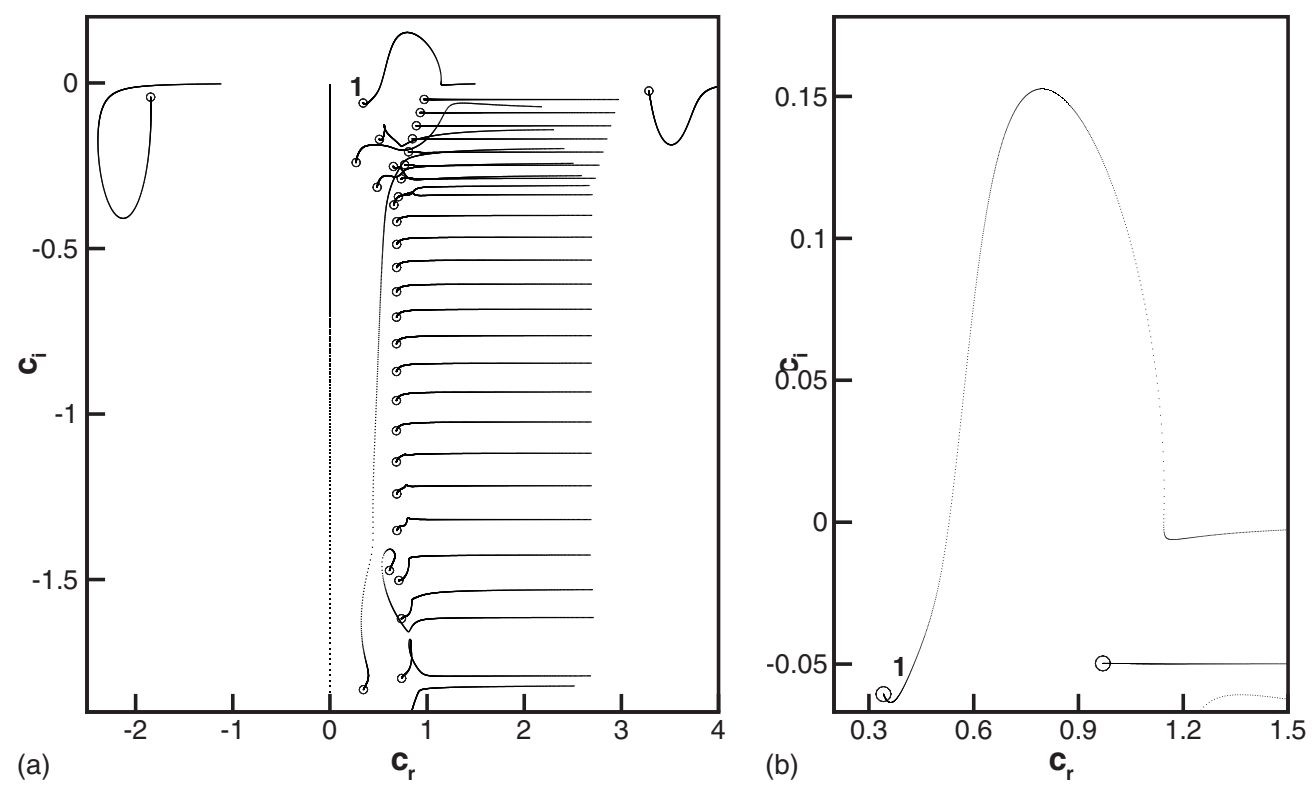

FIG. 13. The trajectories of the phase speeds $c$ for $k=0.5, \mathrm{Re}=10000$ as $\delta$ increases from 0.0001 to 0.01 . The other parameters are $\beta=6^{\prime \prime}, \hat{d}=0.1$, and $\alpha_{B J}=0.1$.

and 0.1 for Aloxite with average size 0.013 or 0.027 in. The values of $\alpha_{B J}$ which we use in the present paper are not intended to be physically realistic; rather, they allow us to characterize the influence of this parameter on the instability of the system. So, our computations also include some results of rather small values of $\alpha_{B J}<0.1$. We note that these small values of $\alpha_{B J}$ may be physically unrealistic.

Physically speaking, a larger $\alpha_{B J}$ corresponds a larger interfacial stress or a smaller interfacial velocity discontinuity. In most of theoretical works on the problem of thermal convective instability, $\alpha_{B J}$ is assumed to have a moderate value 0.1 and the effect of this coefficient has not been taken into account. Recently, Chang et al. [18] investigated the effect of the Beavers-Joseph coefficient on the instability of Poiseuille flow in a fluid-porous system. In this paper, examining effects of $\alpha_{B J}$ is helpful to understand the effect of interfacial shear stress on the different instability modes.

In Fig. 14(a), the marginal curves for various $\alpha_{B J}$ are shown for $\beta=1^{\circ}, \hat{d}=1.0$, and $\delta=0.001$. In this figure, for each $\alpha_{B J}$ the marginal curves display a bimodal structure, i.e., the surface mode and the shear mode. The increase in $\alpha_{B J}$ slightly stabilizes the surface mode in the long-wave region. In the short wave region, the surface mode is insensitive to $\alpha_{B J}$. For a small Beavers-Joseph constant $\alpha_{B J} \leq 0.1$, the shear mode obviously becomes more unstable with the increase in $\alpha_{B J}$. For a large $\alpha_{B J}$, the shear mode is only slightly destabilized with the increase in $\alpha_{B J}$. For example, as shown in Fig. 14(a) the shear branch of $\alpha_{B J}=0.2$ is almost indistinguishable from that of $\alpha_{B J}=0.5$. It seems that the increase in shear stress at the interface enhances the instability of the shear mode, but when $\alpha_{B J}$ exceed 0.2 , the shear mode instability is insensitive to $\alpha_{B J}$. This result is similar to that
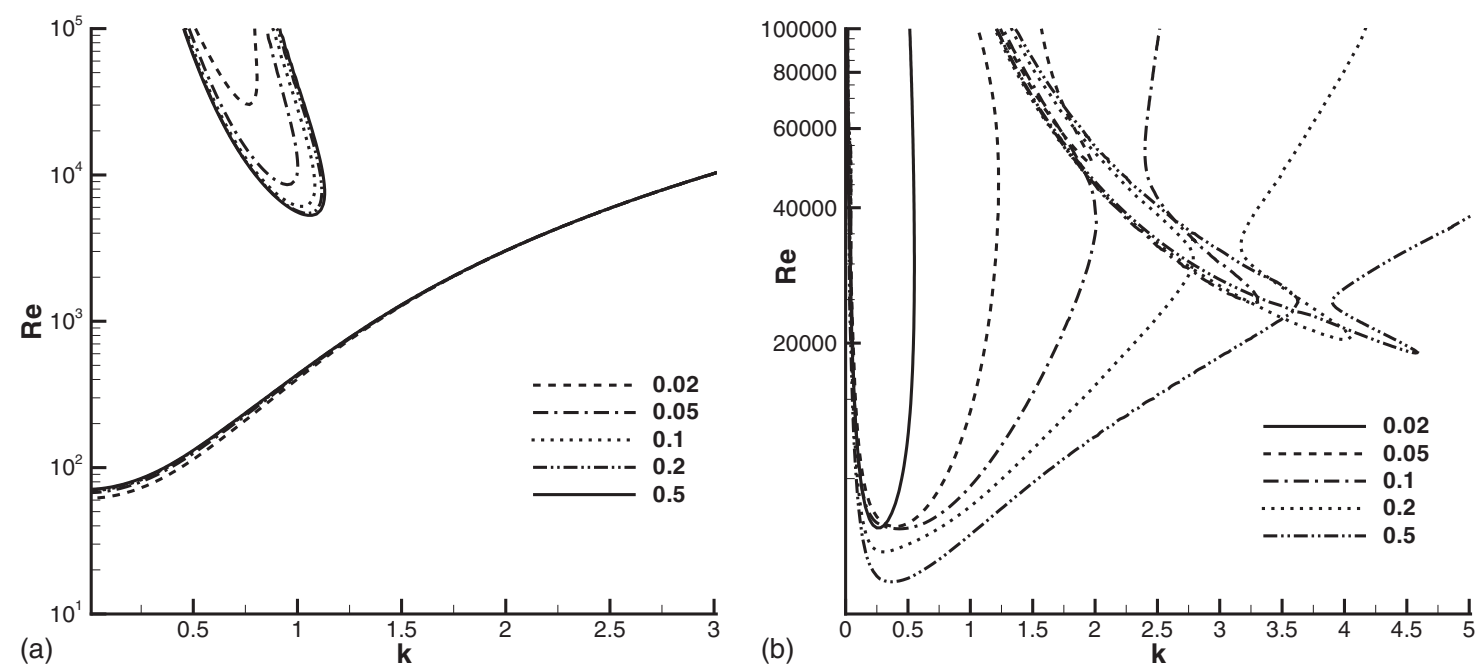

FIG. 14. The marginal curves for various $\alpha_{B J}$ with $\delta=0.001$. (a) $\beta=1^{\circ}, \hat{d}=1.0$; (b) $\beta=6^{\prime \prime}, \hat{d}=0.1$. 

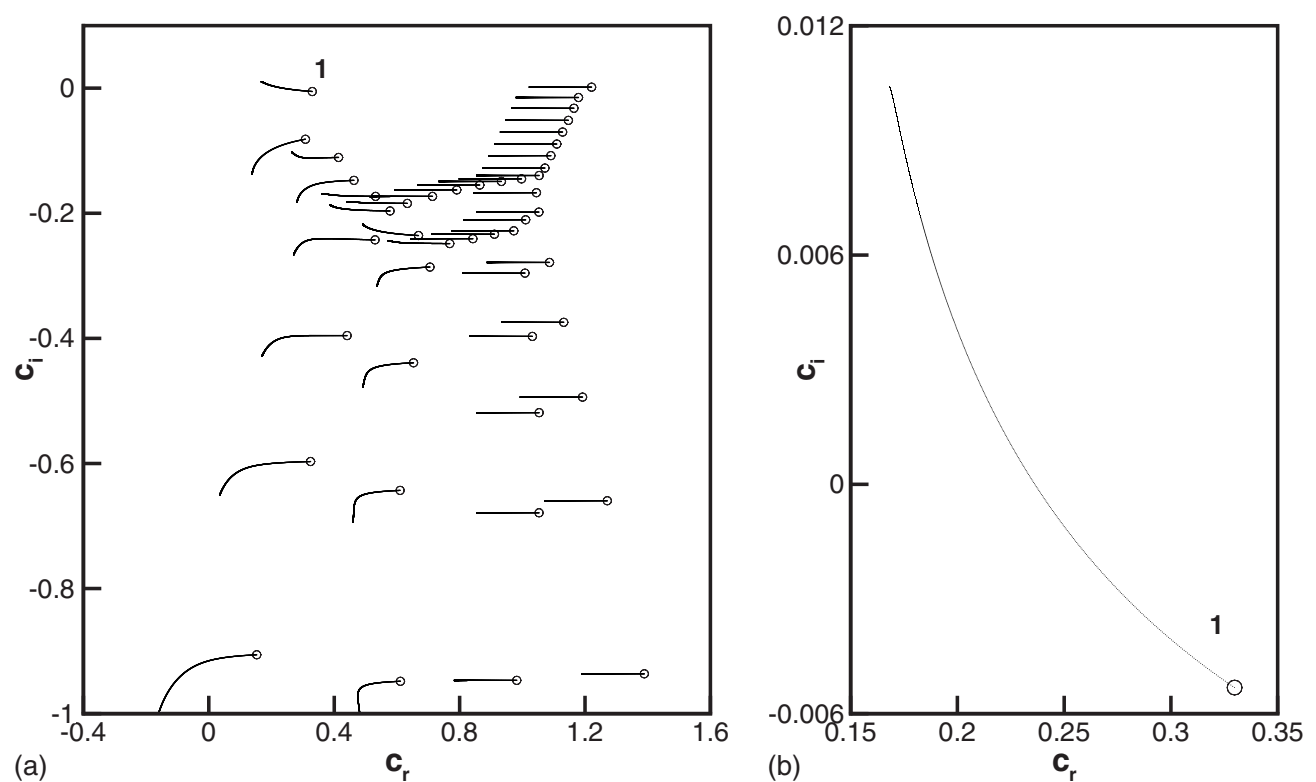

FIG. 15. The trajectories of the phase speeds $c$ for $k=0.75, \operatorname{Re}=30000$ as $\alpha_{B J}$ increases from 0.01 to 1.0 . The other parameters $\beta=1^{\circ}$, $\hat{d}=1.0$, and $\delta=0.001$.

of the problem of Poiseuille flow in a fluid-porous system in the work of Chang et al. [9]. In Fig. 5 in [9], the middle branch of $\delta=0.2$ is only slightly higher than that of $\delta=0.3$, but is apparently lower than that of $\delta=0.1$. However, we should note that the shear mode in our work is not the evenfluid mode in [9].

Figure 15 illustrates the trajectories of the spectrums of different modes as $\alpha_{B J}$ from 0.01 to 1.0 for $k=0.75$, $\operatorname{Re}=30000, \beta=1^{\circ}$, and $\hat{d}=1.0$. The circled points correspond the values of $\alpha_{B J}=0.01$. The trajectory labeled 1 is the shear mode. In Fig. 15, we found it is interesting that $\alpha_{B J}$ only influences $c_{r}$ of the modes on the right branch. However, the imaginary parts $c_{i}$ of the trajectories on the right branch are insensitive to $\alpha_{B J}$. With the increases in $\alpha_{B J}, c_{i}$ of the shear mode increases and this mode becomes an unstable mode. This fact is consistent with the result for Fig. 14(a) that the increase in shear stress at the fluid-porous interface destabilizes the shear mode.

In Fig. 14(b), the marginal curves for various $\alpha_{B J}$ are presented for $\beta=6^{\prime \prime}, \hat{d}=0.1$, and $\delta=0.001$. For a small Beavers-Joseph constant $\alpha_{B J}=0.02$, in the range of $\operatorname{Re} \leq 10^{5}$ only the porous branch presents in the $k-$ Re plane. We believe the reason is that for small $\alpha_{B J}$, the shear stress is not enough to trigger the shear mode; as a result, the porous mode is more unstable than the shear mode. When $\alpha_{B J}$ increases to 0.05 , a shear branch begins to enter in the short wave region. With the increase in $\alpha_{B J}$, the shear mode becomes more unstable. This result indicates that the shear stress of the interface triggers and destabilizes the shear mode. In Fig. 14(b), for small $\alpha_{B J} \leq 0.1$ the increase in $\alpha_{B J}$ only slightly destabilizes porous mode. For $\alpha_{B J} \geq 0.1$, the increase in $\alpha_{B J}$ significantly destabilizes the porous mode. This result indicates that the shear stress at the interface makes the flow more easily penetrate into the porous layer; as a result, the increase in $\alpha_{B J}$ destabilizes the porous mode. In Fig. 14(b), we note that with the increase in $\alpha_{B J}$ both the porous branch and the shear branch extend in the $k-\mathrm{Re}$ plane and intersect in the medium wave number region. In the narrow region between these two modes, both modes are unstable. When the instability develops into nonlinear stage, the interaction of these two modes may be very complicated. However, the nonlinear instability is beyond the scope of this work. In order to understand how $\alpha_{B J}$ influences the instability behaviors of different modes in Fig. 14(b), we present the trajectories of spectrums of different modes as $\alpha_{B J}$ increases from 0.01 to 1.0 for $k=1.0, \operatorname{Re}=20000, \beta=6^{\prime \prime}$, and $\delta=0.001$ in Fig. 16. The circled points correspond to the value of $\alpha_{B J}=0.01$. The trajectory labeled 1 is the porous

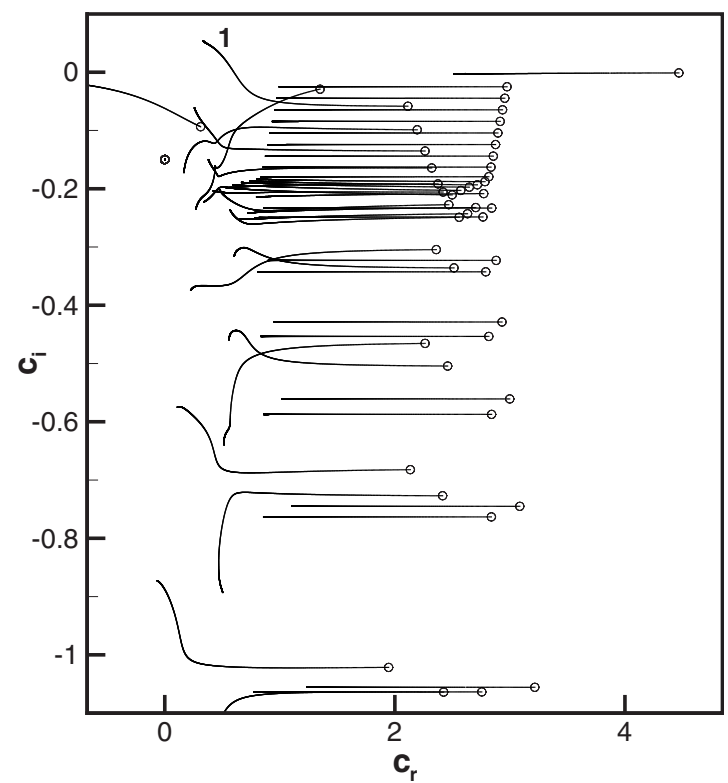

FIG. 16. The trajectories of the phase speeds $c$ for $k=1.0$, $\mathrm{Re}=20000$ as $\alpha_{B J}$ increases from 0.01 to 1.0. The other parameters $\beta=6^{\prime \prime}, \hat{d}=0.1$, and $\delta=0.001$. 
mode. We note that for most modes $c_{r}$ significantly decreases with the increase in $\alpha_{B J}$. For small value of $\alpha_{B J}, c_{i}$ of the porous mode almost maintains a constant. In other words, the instability of porous mode is insensitive to small $\alpha_{B J}$. When $\alpha_{B J}$ is large, $c_{i}$ of the porous mode significantly increases with $\alpha_{B J}$.

Results of Figs. 14-16 conclude that a larger $\alpha_{B J}$ destabilizes both the shear mode and the porous mode. But we should note that the shear mode is sensitive to small $\alpha_{B J}$ and the porous mode is sensitive to large $\alpha_{B J}$. Explanations are given in the following. The increase in $\alpha_{B J}$ has a dual effect on the basic flow. As discussed in the work of Chang et al. [9], with the increase in $\alpha_{B J}$ the shear stress increases at the fluid-porous interface. On the other hand, the increase in $\alpha_{B J}$ results in a deeper penetration of the flow into the porous layer. For a small $\alpha_{B J}$, it is easy to understand that the increase in shear stress destabilizes the fluid mode instability. When $\alpha_{B J}$ is small, because of the resistance of porous medium a small shear stress is not enough to drive the flow to penetrate into the porous layer. As a result, the increase in a small $\alpha_{B J}$ only influences the shear mode. When $\alpha_{B J}$ is large, a larger shear stress makes the flow penetrate deeply into the porous medium. So, the porous mode can more easily occur. At this stage, the increase in $\alpha_{B J}$ mainly influences the penetration thickness rather than the velocity gradient at the interface. As a result, the shear mode becomes insensitive to $\alpha_{B J}$.

\section{CONCLUSIONS}

In the present paper, we have studied the linear stability of a liquid flowing over a porous layer. The Darcy model together with Beavers-Joseph condition is used to describe the flow in the porous layer. We give up the assumption in which the filtration at the interface is neglected and propose a twosided model rather than the one-sided model used in previous works. We compare our results of the two-sided model with that of the one-sided model. For the surface mode instability, we obtain a satisfactory agreement between these two models.

The influences of key parameters $\hat{d}, \delta$, and $\alpha_{B J}$ on the stability are studied in detail in the present paper. For the system in this paper, there are three instability modes, i.e., the surface mode, the shear mode, and the porous mode. The influences of $\hat{d}, \delta$, and $\alpha_{B J}$ on the surface mode can be esti- mated by the combination of these parameters $\beta^{\prime}=\delta / \alpha_{B J} \hat{d}$. For other instability mode, the influences of each parameter are studied respectively.

The influences of $\hat{d}$ on the instabilities of the shear mode are somewhat complicated. With the decrease in $\hat{d}$ from a large value, the marginal curve of the shear mode expands in the $k-\mathrm{Re}$ plane and the shear mode becomes more unstable. As $\hat{d}$ decreases more, the marginal curve of shear mode shrinks in the $k-\mathrm{Re}$ plane and this mode becomes more stable. For a system in which the surface mode is dominant, increase in the Reynolds number may switch the surface mode to the shear mode. For the system with a small inclined angle $\beta$, a porous mode is found for small $\hat{d}$. With the decrease in $\hat{d}$, the porous mode becomes more unstable. The results in this paper show that the increase in Re may switch the porous mode to the shear mode. We should note that the physical parameters $\delta=10^{-2}-10^{-4}$ and $\alpha_{B J}=0.1-1.0$ are in realistic range, so transitions between different instability modes can be encountered in physically realistic porous-fluid systems.

The permeability plays an important role in determining the instability of both the shear mode and the porous mode. For small $\delta$, the increase in $\delta$ will destabilize the shear mode. For large $\hat{d}$, with the increase in $\delta$ the shear mode becomes more stable. The porous mode becomes more unstable with the increase in $\delta$ because the flow can more easily penetrate into the porous medium with a higher permeability.

We also investigate the influence of $\alpha_{B J}$ on the instability in detail. The increase in $\alpha_{B J}$ has a dual effect, i.e., increasing of the shear stress at the interface and increasing the penetration thickness into the porous layer. The former prevails in the case of small $\alpha_{B J}$ and the latter in the case of large $\alpha_{B J}$. As a result, the increase in $\alpha_{B J}$ destabilizes both the shear mode and the porous mode. However, the shear mode is sensitive to small $\alpha_{B J}$ and the porous mode is sensitive to large $\alpha_{B J}$.

\section{ACKNOWLEDGMENTS}

The authors thank the financial support for this research from National Foundation of China (Grants No. 10772185 and No. 50890182) and the Knowledge Innovation Program of Chinese Academy of Sciences (Grant No. KGCX-SW409).
[1] T. B. Benjamin, J. Fluid Mech. 2, 554 (1957).

[2] C. S. Yih, Phys. Fluids 6, 321 (1963).

[3] G. J. De Bruin, J. Eng. Math. 8, 259 (1974).

[4] S. P. Lin, Phys. Fluids 10, 308 (1967).

[5] J. M. Floryan, S. H. Davis, and R. E. Kelly, Phys. Fluids 30, 983 (1987).

[6] F. Chen and C. F. Chen, J. Heat Transfer 110, 403 (1988).

[7] T. Desaive, G. Lebon, and M. Hennenberg, Phys. Rev. E 64, 066304 (2001).
[8] D. A. Nield and A. Bejan, Convection in Porous Media, 2nd ed. (Springer, New York, 1999).

[9] M. H. Chang, F. Chen, and B. Straughan, J. Fluid Mech. 564, 287 (2006).

[10] G. S. Beavers and D. D. Joseph, J. Fluid Mech. 30, 197 (1967).

[11] A. A. Hill and B. Staughan, J. Fluid Mech. 603, 137 (2008).

[12] R. Liu, Q. S. Liu, and S. C. Zhao, Phys. Fluids 20, 104105 (2008). 
[13] J. P. Pascal, J. Phys. D 32, 417 (1999).

[14] J. P. Pascal, J. Non-Newtonian Fluid Mech. 133, 109 (2006).

[15] I. M. R. Sadiq and R. Usha, Phys. Fluids 20, 022105 (2008).

[16] P. J. Schmid and D. S. Henningson, Stability and Transition in Shear Flows (Springer-Verlag, New York, 2001).
[17] M. A. Combarnous and S. A. Bories, Adv. Hydrosci. 10, 231 (1975).

[18] M. H. Chang, Phys. Fluids 18, 035104 (2006).

[19] L. S. Tuckerman, J. Comput. Phys. 80, 403 (1989). 